\title{
The nonlinear Schrödinger equation in the half-space
}

\author{
Antonio J. Fernández ${ }^{1} \cdot$ Tobias Weth $^{2}$ \\ Received: 21 August 2020 / Revised: 24 November 2020 / Accepted: 6 December 2020 / \\ Published online: 16 January 2021 \\ (C) The Author(s) 2021
}

\section{Abstract}

The present paper is concerned with the half-space Dirichlet problem

$$
\begin{array}{ll}
-\Delta v+v=|v|^{p-1} v, & \text { in } \mathbb{R}_{+}^{N}, \\
v=c, \text { on } \partial \mathbb{R}_{+}^{N}, & \lim _{N} v\left(x^{\prime}, x_{N}\right)=0 \text { uniformly in } x^{\prime} \in \mathbb{R}^{N-1},
\end{array}
$$

where $\mathbb{R}_{+}^{N}:=\left\{x \in \mathbb{R}^{N}: x_{N}>0\right\}$ for some $N \geq 1$ and $p>1, c>0$ are constants. We analyse the existence, non-existence and multiplicity of bounded positive solutions to $\left(P_{c}\right)$. We prove that the existence and multiplicity of bounded positive solutions to $\left(P_{c}\right)$ depend in a striking way on the value of $c>0$ and also on the dimension $N$. We find an explicit number $c_{p} \in(1, \sqrt{e})$, depending only on $p$, which determines the threshold between existence and non-existence. In particular, in dimensions $N \geq 2$, we prove that, for $0<c<c_{p}$, problem $\left(P_{c}\right)$ admits infinitely many bounded positive solutions, whereas, for $c>c_{p}$, there are no bounded positive solutions to $\left(P_{c}\right)$.

\section{Introduction}

Due to its relevance within several models arising in physics and biology, the nonlinear stationary Schrödinger equation

$$
-\Delta v+v=|v|^{p-1} v, \quad \text { in } \mathbb{R}^{N}
$$

Communicated by Y. Giga.

Tobias Weth

weth@math.uni-frankfurt.de

Antonio J. Fernández

ajf77@bath.ac.uk

1 Department of Mathematical Sciences, University of Bath, Bath BA2 7AY, UK

2 Institut für Mathematik, Goethe-Universität Frankfurt, Robert-Mayer-Str. 10, 60629 Frankfurt am Main, Germany 
received extensive attention in the last four decades. In particular, let us mention that the study of solitary wave solutions for the (focusing) NLS

$$
i \partial_{t} \varphi+\Delta \varphi+|\varphi|^{p-1} \varphi=0, \quad(t, x) \in \mathbb{R} \times \mathbb{R}^{N},
$$

is reduced to problem (1.1) via a time-harmonic ansatz. For classic existence and multiplicity results, we refer the reader e.g. to the seminal papers $[2,3,6,8,9,30,31]$ and the monographs [32,34]. We also recall the fundamental works [16,24] where the radial symmetry and uniqueness, up to translations, of positive solutions to (1.1) satisfying the decay condition

$$
v(x) \rightarrow 0, \quad \text { as }|x| \rightarrow \infty,
$$

are proved in the case $1<p<2^{*}-1$. Here and in the following $2^{*}$ denotes the critical Sobolev exponent, i.e. $2^{*}=\frac{2 N}{N-2}$ for $N \geq 3$ and $2^{*}=2^{*}-1=\infty$ for $N=1,2$. In particular, these results imply the uniqueness, up to translations, of positive finite energy solutions $u \in H^{1}\left(\mathbb{R}^{N}\right)$. In contrast, for $N \geq 2$, (1.1) admits an abundance of sign-changing finite energy solutions satisfying (1.2), see e.g. $[2,3,27,28]$ and the references therein. Moreover, more recent geometric constructions of different solution shapes highlight the rich structure of the set of positive solutions which do not satisfy the decay assumption (1.2), see e.g. $[1,11,26,28]$ and the references therein.

Whereas it seems impossible to provide an exhaustive list of references for the full space problem (1.1), much less is known regarding the half-space Dirichlet problem

$$
\left\{\begin{aligned}
-\Delta v+v & =|v|^{p-1} v, & & \text { in } \mathbb{R}_{+}^{N}, \\
v & =c, & & \text { on } \partial \mathbb{R}_{+}^{N},
\end{aligned}\right.
$$

where $\mathbb{R}_{+}^{N}:=\left\{x \in \mathbb{R}^{N}: x_{N}>0\right\}$ for some $N \geq 1$ and $c \geq 0$ is a constant. In the case $c=0$, general nonexistence results are available for (1.3). More precisely, the non-existence of finite energy solutions $u \in H_{0}^{1}\left(\mathbb{R}_{+}^{N}\right)$ to (1.3) in the case $c=0$ follows from [14, Theorem I.1], while [4, Corollary 1.3] yields, in particular, the non-existence of positive solutions to (1.3) with $c=0$ and the the decay property

$$
\lim _{x_{N} \rightarrow \infty} v\left(x^{\prime}, x_{N}\right)=0, \quad \text { uniformly in } x^{\prime} \in \mathbb{R}^{N-1} .
$$

The aim of the present paper is to analyse the existence, non-existence and multiplicity of bounded positive solutions $v$ to the problem (1.3)-(1.4) in the case $c>0$, for which we are not aware of any previous result in general dimensions $N$. As we shall see below, the multiplicity of positive solutions depends in a striking way on the value $c>0$ and, somewhat surprisingly, also on the dimension $N$. Let us stress that we cannot expect the existence of finite energy solutions $u \in H^{1}\left(\mathbb{R}_{+}^{N}\right)$ to (1.3) in the case $N \geq 2$. Actually, we cannot expect solutions to (1.3) belonging to $L^{p}\left(\mathbb{R}_{+}^{N}\right)$ for any $1 \leq p<\infty$. The one-dimensional decay condition (1.4) therefore seems natural.

The consideration of inhomogeneous Dirichlet boundary conditions as in (1.3) is to some extend motivated by recent works on pattern formation in biological and 
chemical models. For instance, in [23], the authors numerically show that, for different types of reaction-diffusion systems, the pattern formation can be isolated away from the boundary using this type of boundary conditions. Moreover, within a rigorous analysis of some of these models in an asymptotically small diffusivity ratio, one may expect that the equation of the limiting profile is precisely (1.3) with $c>0$. This is indeed the case for the Gierer-Meinhardt system considered in [19]. As one can observe in [19, Sect. 2], the homoclinic solution $w_{0}\left(\cdot+t_{c, p}\right)$ (see (1.6) and (1.7) below) plays a key role in the construction of multi-spike patterns for this model.

Not surprisingly, problem (1.3) is completely understood in the case $N=1$. This is due to the fact that the one-dimensional equation

$$
-w^{\prime \prime}+w=w^{p}
$$

admits a first integral, and from this one can easily deduce that, for $1<p<\infty$, (1.5) admits, up to sign and translation, a unique global non trivial solution satisfying $w(t) \rightarrow 0$ as $t \rightarrow \pm \infty$. See e.g. [8, Theorem 5], [34, Theorem 3.16] and [22, Theorem 1.2] where, in addition, the Mountain-Pass characterization of this unique solution is established. By direct computations, one can verify that this solution is precisely given by

$$
\begin{aligned}
& t \mapsto w_{0}(t)=c_{p}\left[\cosh \left(\frac{p-1}{2} t\right)\right]^{-\frac{2}{p-1}}, \\
& \text { with } \quad c_{p}:=\left(\frac{p+1}{2}\right)^{\frac{1}{p-1}}=w_{0}(0)=\sup _{t \in \mathbb{R}} w_{0}(t) .
\end{aligned}
$$

As we shall see below, the value $c_{p}$ will be of key importance also for the higher dimensional version of (1.3). The following complete characterization of the one dimensional case is an immediate consequence of these facts.

Proposition 1.1 Let $N=1, p>1$ and $c>0$. Then:

(i) If $0<c<c_{p}$, problem (1.3)-(1.4) admits exactly two positive solutions given by $t \mapsto w_{0}\left(t+t_{c, p}\right)$ and $t \mapsto w_{0}\left(t-t_{c, p}\right)$, where

$$
t_{c, p}:=\frac{2}{p-1} \ln \left(\sqrt{\frac{p+1}{2 c^{p-1}}}+\sqrt{\frac{p+1}{2 c^{p-1}}-1}\right) .
$$

(ii) If $c=c_{p}$, the function $w_{0}$ is the unique positive solution to (1.3)-(1.4).

(iii) If $c>c_{p}$, problem (1.3)-(1.4) does not admit solutions.

Our main results concern dimensions $N \geq 2$. In this case, problem (1.3)-(1.4) is invariant under translations and rotations parallel to the boundary $\partial \mathbb{R}_{+}^{N}=\mathbb{R}^{N-1}$. In particular, if $N \geq 2$ and $v$ is a positive solution to (1.3)-(1.4), then the functions $x \mapsto v\left(x^{\prime}+\tau, x_{N}\right), \tau \in \mathbb{R}^{N-1}$ are also solutions to (1.3)-(1.4), where, here and in the following, we write $x=\left(x^{\prime}, x_{N}\right)$ for $x \in \mathbb{R}_{+}^{N}$ with $x^{\prime} \in \mathbb{R}^{N-1}$. In the following, 
we call two solutions geometrically distinct if they do not belong to the same orbit of solutions under translations and rotations in $\mathbb{R}^{N-1}$.

Our first main result reads as follows.

Theorem 1.2 Let $N \geq 2, p>1$ and $c>0$. Then:

(i) If $0<c<c_{p}$, there exist at least three geometrically distinct bounded positive solutions to (1.3)-(1.4).

(ii) If $c>c_{p}$, there are no bounded positive solutions to (1.3)-(1.4).

Remark 1.1 (a) By a bounded positive solution to (1.3)-(1.4), we mean a positive function $v \in C^{2}\left(\mathbb{R}_{+}^{N}\right) \cap C\left(\overline{\mathbb{R}_{+}^{N}}\right) \cap L^{\infty}\left(\mathbb{R}_{+}^{N}\right)$ satisfying (1.3) in the pointwise sense and such that (1.4) holds.

(b) The nonexistence part (ii) of Theorem 1.2 is proved with a variant of the sliding method based on a comparison with $x_{N}$-translates of the function $x \mapsto w_{0}\left(x_{N}\right)$. We shall comment on this in more detail further below.

(c) As we have indicated above already, Theorem 1.2 (i) highlights the rich structure of solutions to (1.3)-(1.4) in the case $N \geq 2,0<c<c_{p}$, which is in striking contrast to the case $N=1$. Indeed, Theorem 1.2 (i) yields, in addition to the two onedimensional profile solutions $x \mapsto w_{0}\left(x_{N}+t_{c, p}\right)$ and $x \mapsto w_{0}\left(x_{N}-t_{c, p}\right)$ at least one further geometrically distinct solution which is not merely a function of the $x_{N}$-variable. Hence, by the remarks above, this one solution gives rise to an infinite number of positive solutions via translations parallel to the boundary $\partial \mathbb{R}_{+}^{N}=$ $\mathbb{R}^{N-1}$. In Corollary 1.4 below, we shall derive more precise lower estimates on the number of geometrically distinct solutions depending on the exponent $p$ and the dimension $N$.

(d) It suffices to prove Theorem 1.2 (i) in the case $N=2$ since every positive solution $v$ to (1.3)-(1.4) in the case $N=2$ gives rise to a corresponding solution $\tilde{v}$ to (1.3)(1.4) in general dimension $N \geq 3$ by simply setting $\tilde{v}(x)=v\left(x_{1}, x_{N}\right)$.

(e) It remains as an interesting open question whether the function $x \mapsto w_{0}\left(x_{N}\right)$ is the unique bounded positive solution to (1.3)-(1.4) in the case $c=c_{p}$. At first glance, it seems natural to establish such a uniqueness result also with the help of a sliding argument as mentioned in (b) above, but additional difficulties appear in the case $c=c_{p}$, and non-uniqueness remains a possibility for now.

The following result provides some information on the shape of the solutions we construct.

Theorem 1.3 Let $N \geq 2,1<p<2^{*}-1$ and $0<c<c_{p}$. Then there exists a positive solution to (1.3)-(1.4) of the form

$$
x \mapsto w_{0}\left(x_{N}+t_{c, p}\right)+u(x),
$$

with a nonnegative function $u \in H_{0}^{1}\left(\mathbb{R}_{+}^{N}\right) \backslash\{0\}$.

By the remarks above and since all exponents $p<\infty$ are subcritical in the case $N=2$, Theorem 1.3 implies Theorem 1.2 (i) in the case $N=2$ and therefore for all $N \geq 2$. It also allows us to distinguish different solution orbits under translations and rotations in $\mathbb{R}^{N-1}$. 
Corollary 1.4 Let $N \geq 3,1<p<\frac{M+2}{M-2}$ for some $M \in\{3, \ldots, N\}$ and $0<c<$ $c_{p}$. Then problem (1.3)-(1.4) admits at least $M+1$ geometrically distinct positive solutions.

This result is a rather immediate corollary of Theorem 1.3. Indeed, under the given assumptions, for every dimension $\widetilde{N} \in\{2,3, \ldots, M\}$, Theorem 1.3 yields the existence of a solution to (1.3)-(1.4) of the form

$$
x \mapsto w_{0}\left(x_{\tilde{N}}+t_{c, p}\right)+u\left(x_{1}, \ldots, x_{\tilde{N}-1}, x_{\tilde{N}}\right)
$$

with a nonnegative $u \in H_{0}^{1}\left(\mathbb{R}_{+}^{\tilde{N}}\right) \backslash\{0\}$. Clearly, these $M-1$ solutions are geometrically distinct, and they are also geometrically distinct from the two one-dimensional profile solutions.

It is natural to guess that the change of the solution set when passing from $c>c_{p}$ to $c<c_{p}$ is a bifurcation phenomenon. More precisely, one may guess that the solutions constructed in Theorem 1.3 have the property that $u=u_{c} \rightarrow 0 \in H_{0}^{1}\left(\mathbb{R}_{+}^{N}\right)$ as $c \nearrow c_{p}$ for the functions $u$ in the ansatz (1.8). This remains an open question, and the answer could even depend on the value of $p$. We note that standard results from bifurcation theory do not apply here since the linearized problem

$$
\left\{\begin{array}{rr}
-\Delta v+v-p\left|w_{0}\left(x_{N}\right)\right|^{p-1} v=0 & \text { in } \mathbb{R}_{+}^{N}, \\
v & =0
\end{array} \quad \text { on } \partial \mathbb{R}_{+}^{N},\right.
$$

at the parameter value $c=c_{p}$ has purely essential spectrum due to its invariance with respect to translations in directions parallel to the boundary $\partial \mathbb{R}_{+}^{N}=\mathbb{R}^{N-1}$. Bifurcation from the essential spectrum has been observed successfully in other contexts (see e.g. the survey paper [33] and the references therein), but there is still no general functional analytic framework which provides sufficient abstract conditions.

We now give some ideas of the proof of Theorem 1.3. For this we fix $c \in\left(0, c_{p}\right)$ and define the functions

$$
t \mapsto z_{c}(t):=w_{0}\left(t+t_{c, p}\right) \quad \text { and } \quad t \mapsto \widetilde{z}_{c}(t):=w_{0}\left(t-t_{c, p}\right)
$$

where $t_{c, p}$ is given in (1.7). We recall that $z_{c}$ and $\widetilde{z}_{c}$ are the unique positive solutions to $(1.5)$ such that $z_{c}(0)=\widetilde{z}_{c}(0)=c$. Moreover, we define $u_{c}: \overline{\mathbb{R}_{+}^{N}} \rightarrow \mathbb{R}$ and $\tilde{u}_{c}: \overline{\mathbb{R}_{+}^{N}} \rightarrow \mathbb{R}$ as

$$
u_{c}(x):=z_{c}\left(x_{N}\right) \quad \text { and } \quad \tilde{u}_{c}(x):=\widetilde{z}_{c}\left(x_{N}\right)
$$

and we directly notice that $u_{c}$ and $\tilde{u}_{c}$ are both solutions to (1.3)-(1.4). Furthermore, it follows that

$$
u_{c}(x)=O\left(e^{-x_{N}}\right) \quad \text { and } \quad \tilde{u}_{c}(x)=O\left(e^{-x_{N}}\right), \quad \text { as } x_{N} \rightarrow \infty .
$$


Proving Theorem 1.3 now amounts to find a nonnegative solution $u \in H_{0}^{1}\left(\mathbb{R}_{+}^{N}\right) \backslash\{0\}$ to the non-autonomous Schrödinger type equation

$$
-\Delta u+u=f(x, u), \quad u \in H_{0}^{1}\left(\mathbb{R}_{+}^{N}\right),
$$

with

$$
f(x, s):=\left|u_{c}(x)+s\right|^{p-1}\left(u_{c}(x)+s\right)-\left(u_{c}(x)\right)^{p},
$$

because in this case $v=u_{c}+u$ is of the form (1.8), solves (1.3) and it is easy to see that also satisfies (1.4). Since we are interested in finding non-negative solutions to (1.11), we truncate the nonlinearity and define

$$
g(x, s):=\left(u_{c}(x)+s^{+}\right)^{p}-\left(u_{c}(x)\right)^{p}= \begin{cases}f(x, s), & \text { if } s \geq 0, \\ 0, & \text { if } s \leq 0,\end{cases}
$$

with $f$ given in (1.12). We then consider the auxiliary problem

$$
-\Delta u+u=g(x, u), \quad u \in H_{0}^{1}\left(\mathbb{R}_{+}^{N}\right) .
$$

Considering $u^{-} \in H_{0}^{1}\left(\mathbb{R}_{+}^{N}\right)$ as test function in (1.14), one can easily check that every solution to (1.14) is non-negative and so, that every solution to (1.14) is a non-negative solution to (1.11). It might be worth pointing out that the one-dimensional function $\tilde{u}:=\tilde{u}_{c}-u_{c} \in C^{2}\left(\mathbb{R}_{+}^{N}\right)$ is a positive solution to the equation in (1.14) and also satisfies $\tilde{u}=0$ on $\partial \mathbb{R}_{+}^{N}$. However, $\tilde{u} \notin H_{0}^{1}\left(\mathbb{R}_{+}^{N}\right)$ since it only depends on the $x_{N}$ variable. Hence, $\tilde{u}$ is not a solution to (1.14).

We shall look for a non-trivial solution to (1.14) as a critical point of the associated functional

$$
E: H_{0}^{1}\left(\mathbb{R}_{+}^{N}\right) \rightarrow \mathbb{R}, \quad E(u)=\frac{1}{2} \int_{\mathbb{R}_{+}^{N}}\left(|\nabla u|^{2}+u^{2}\right) d x-\int_{\mathbb{R}_{+}^{N}} G(x, u) d x,
$$

where

$$
G(x, u):=\int_{0}^{u} g(x, s) d s=\frac{1}{p+1}\left(\left(u_{c}+u^{+}\right)^{p+1}-u_{c}^{p+1}-(p+1) u_{c}^{p} u^{+}\right) .
$$

More precisely, we are going to prove the existence of a non-trivial critical point of mountain pass type. This requires new and subtle estimates. The key difficulties in the variational approach are the non-standard shape of the nonlinearity $g$ in (1.14) and the lack of compactness due to the unboundedness of $\mathbb{R}_{+}^{N}$. To overcome these difficulties, we need new estimates within the analysis of Cerami sequences and for comparing the mountain pass energy value for $E$ with the corresponding one of the limit energy functional

$$
E_{\infty}: H^{1}\left(\mathbb{R}^{N}\right) \rightarrow \mathbb{R}, \quad E_{\infty}(u)=\frac{1}{2} \int_{\mathbb{R}^{N}}\left(|\nabla u|^{2}+u^{2}\right) d x-\frac{1}{p+1} \int_{\mathbb{R}^{N}}|u|^{p+1} d x .
$$


In particular, we shall use the asymptotic decay properties of the unique positive radial solution to (1.1) in order to build suitable test functions.

We wish to mention two further open problems at this stage. First, one may ask whether Theorem 1.3 extends to the critical case $N \geq 3, p=2^{*}-1$. In this case, the mountain pass geometry of the functional $E$ remains, but the lack of compactness is more severe as it is not only caused by the unboundedness of $\mathbb{R}_{+}^{N}$ but also by possible point concentration of bounded Cerami sequences. Indeed, while the limit energy functional $E_{\infty}$ in (1.17) does not admit critical points in the case $p=2^{*}$ by Pohozaev's identity (see e.g. [34, Corollary B.4]), rescaling bounded Cerami sequences around possible concentration points leads to critical points of the Yamabe functional $u \mapsto$ $\frac{1}{2} \int_{\mathbb{R}^{N}}|\nabla u|^{2} d x-\frac{1}{2^{*}} \int_{\mathbb{R}^{N}}|u|^{2^{*}} d x$. It therefore seems natural to build test functions from translated and concentrated instantons $x \mapsto\left[N(N-2) \varepsilon^{2}\right]^{\frac{N-2}{4}}\left(\varepsilon^{2}+\left|x-x_{*}\right|^{2}\right)^{-\frac{N-2}{2}}$ in order to estimate the mountain pass energy. However, since our estimates rely on the precise exponential decay rate of the unique positive radial solution to (1.1) as given in (4.6), they do not apply to instantons. Hence the case $p=2^{*}$ remains a problem for future research.

The second open problem concerns the existence of solutions of the form $x \mapsto$ $w_{0}\left(x_{N}-t_{c, p}\right)+u(x)$ with $u \in H_{0}^{1}\left(\mathbb{R}_{+}^{N}\right) \backslash\{0\}$. In this case, $u_{c}$ has to be replaced by $\tilde{u}_{c}$ in the definition of the nonlinearity $G$ in (1.16). One may observe that the mountain pass geometry is lost in this case, and the presence of essential spectrum leads to additional difficulties which seem hard to deal with.

We now comment on the proofs of the non-existence part (ii) of Theorem 1.2. We argue by contradiction and use a suitable modification of the so-called sliding method introduced by H. Berestycki and L. Nirenberg and further developed by H. Berestycki, L. Caffarelli and L. Nirenberg among others. Specifically, our proofs are inspired by [15, Sect. 2] and [7, Sect. 4].

We finally comment on the boundedness of positive solutions to (1.3)-(1.4). As stated in the following proposition, all the positive solutions to (1.3)-(1.4) are bounded in the case where $1<p<2^{*}-1$. Hence, the fact that we are considering bounded solutions is not a restriction in this case.

Proposition 1.5 Let $N \geq 2,1<p<2^{*}-1$ and $c>0$. Any positive $v \in C^{2}\left(\mathbb{R}_{+}^{N}\right) \cap$ $C\left(\overline{\mathbb{R}_{+}^{N}}\right)$-solution to $(1.3)-(1.4)$ belongs to $L^{\infty}\left(\mathbb{R}_{+}^{N}\right)$.

The proof of Proposition 1.5 follows by a rather standard blow up argument based on the doubling lemma by P. Poláčik, P. Quittner and P. Souplet in [29]. For the convenience of the reader, we include the proof in Sect. 5 below.

\section{Organization of the paper}

In Sect. 2, we collect estimates related to the nonlinearity $g$ in (1.13) and the functional $E$ associated with (1.14). With the help of these estimates, we establish the mountain pass geometry of $E$ in Sect. 3, and we show that Cerami sequences at nontrivial energy levels are bounded and admit nontrivial weak limits after suitable translation. In Sect. 4, we then prove a key energy estimate which shows that, in dimensions $N \geq 2$, the mountain pass energy of the functional $E$ is strictly smaller than the corresponding 
one for the limit energy functional $E_{\infty}$ given in (1.17). With the help of this energy estimate, we then complete the proof of Theorem 1.3 in Sect. 5. Finally, we give the proof of Theorem 1.2 (ii) in Sect. 6.

\section{Notation}

For $1 \leq p<\infty$, we let $\|\cdot\|_{L^{p}\left(\mathbb{R}_{+}^{N}\right)}$ denote the standard norm on the usual Lebesgue space $L^{p}\left(\mathbb{R}_{+}^{N}\right)$. The Sobolev space $H_{0}^{1}\left(\mathbb{R}_{+}^{N}\right)$ is endowed with the standard norm

$$
\|u\|^{2}=\int_{\mathbb{R}_{+}^{N}}\left(|\nabla u|^{2}+|u|^{2}\right) d x .
$$

Also, for a function $v$, we define $v^{+}:=\max \{v, 0\}$ and $v^{-}:=\max \{-v, 0\}$ and we write $x=\left(x^{\prime}, x_{N}\right)$ for $x \in \mathbb{R}_{+}^{N}$ with $x^{\prime} \in \mathbb{R}^{N-1}$. We denote by ${ }^{\prime} \rightarrow^{\prime}$, respectively by ${ }^{\prime} \rightarrow^{\prime}$, the strong convergence, respectively the weak convergence in corresponding space and denote by $B_{R}(x)$ the open ball in $\mathbb{R}^{N}$ of center $x$ and radius $R>0$. Also, we shall denote by $C_{i}>0$ different constants which may vary from line to line but are not essential to the analysis of the problem. Finally, at various places, we have to distinguish the cases $p \leq 2$ and $p>2$. For this it is convenient to introduce the special constant

$$
1_{\{p>2\}}:= \begin{cases}0, & p \leq 2 \\ 1, & p>2\end{cases}
$$

\section{Preliminaries}

In this section we collect some estimates related to the transformed nonlinearity $g$ defined in (1.13), its primitive $G$ and the functional $E$ defined in (1.15). For this we fix, throughout Sects. $2-5, c \in\left(0, c_{p}\right), p \in\left(1,2^{*}-1\right)$, and we let $u_{c}$ be given in (1.9). We recall that we have the uniform estimate

$$
0 \leq u_{c} \leq c_{p} \quad \text { in } \mathbb{R}_{+}^{N}
$$

We start with an elementary inequality for nonnegative real numbers which will be used in the energy estimates in Sect. 4 below.

Lemma 2.1 For every $q>2$ there exists $\kappa_{q}>0$ with

$$
(a+b)^{q}-a^{q}-b^{q} \geq q a^{q-1} b+\kappa_{q} a b^{q-1} \quad \text { for all } a, b \geq 0 .
$$

Remark 2.1 If $q \geq 3$, then (2.2) holds in symmetric form with $\kappa_{q}=q$, see e.g. [20, Theorem 1]. If $q \in(2,3)$, it is easy to see that one has to choose $\kappa_{q}<q$. 
Proof We first note that, since $q-1>1$, we have, by convexity of the function $\tau \mapsto(1+\tau)^{q-1}$,

$$
\begin{aligned}
(s+t)^{q-1}-\left(s^{q-1}+t^{q-1}\right) & =s^{q-1}\left[\left(1+\frac{t}{s}\right)^{q-1}-\left(1+\left(\frac{t}{s}\right)^{q-1}\right)\right] \\
& \geq s^{q-1}\left((q-1) \frac{t}{s}-\left(\frac{t}{s}\right)^{q-1}\right) \\
& =(q-1) t s^{q-2}-t^{q-1}
\end{aligned}
$$

for $s \geq t>0$. Now, to prove the claim, it suffices to consider $a, b>0$, since the inequality holds trivially if $a=0$ or $b=0$. Moreover, it suffices to prove that the inequality holds for $b \geq a>0$ with some $\kappa_{q} \in(0, q]$, since then it also follows for arbitrary $a, b>0$. For fixed $a>0$, we consider the function

$$
\ell:[0, \infty) \rightarrow \mathbb{R}, \quad \ell(t)=(a+t)^{q}-a^{q}-t^{q}-q a^{q-1} t
$$

Then we have $\ell(0)=0$ and

$$
\ell^{\prime}(t)=q\left[(a+t)^{q-1}-\left(a^{q-1}+t^{q-1}\right)\right]
$$

Consequently, by (2.3) we have, for $b \geq a$,

$$
\begin{aligned}
\ell(b)= & \int_{0}^{a} \ell^{\prime}(t) d t+\int_{a}^{b} \ell^{\prime}(t) d t \geq q\left[\int_{0}^{a}\left((q-1) t a^{q-2}-t^{q-1}\right) d t\right. \\
& \left.+\int_{a}^{b}\left((q-1) a t^{q-2}-a^{q-1}\right) d t\right] \\
= & q a\left(\kappa_{q, 1} a^{q-1}+b^{q-1}-a^{q-2} b\right) \\
& \text { with } \quad \kappa_{q, 1}:=\frac{q-1}{2}-\frac{1}{q}>0 .
\end{aligned}
$$

Since, by Young's inequality,

$$
a^{q-2} b \leq \frac{q-2}{q-1} a^{q-1}+\frac{1}{q-1} b^{q-1},
$$

we deduce that

$$
\ell(b) \geq q a\left[\left(\kappa_{q, 1}-\frac{q-2}{q-1}\right) a^{q-1}+\frac{q-2}{q-1} b^{q-1}\right] .
$$


If $\kappa_{q, 1} \geq \frac{q-2}{q-1}$, we conclude that $\ell(b) \geq \frac{q(q-2)}{q-1} a b^{q-1}$. On the other hand, if $0<$ $\kappa_{q, 1}<\frac{q-2}{q-1}$, we use again that $b \geq a$ and conclude that

$$
\ell(b) \geq q a\left[\left(\kappa_{q, 1}-\frac{q-2}{q-1}\right) b^{q-1}+\frac{q-2}{q-1} b^{q-1}\right]=q \kappa_{q, 1} a b^{q-1} .
$$

Hence, (2.2) holds for $b \geq a>0$ with $\kappa_{q}=q \min \left\{\frac{q-2}{q-1}, \kappa_{q, 1}\right\} \in(0, q)$. The proof is finished.

Next we provide basic but important estimates for the nonlinearity $g$ defined in (1.13) and its primitive $G$.

\section{Lemma 2.2}

(i) $\operatorname{For}(x, s) \in \mathbb{R}_{+}^{N} \times \mathbb{R}$ we have

$$
0 \leq g(x, s)-s^{+} p u_{c}(x)^{p-1} \leq C_{1, p}\left[s^{+}\right]^{p}+1_{\{p>2\}} C_{2, p}\left[s^{+}\right]^{2},
$$

and

$$
0 \leq G(x, s)-\frac{p}{2}\left[s^{+}\right]^{2} u_{c}(x)^{p-1} \leq \frac{C_{1, p}}{p+1}\left[s^{+}\right]^{p+1}+1_{\{p>2\}} \frac{C_{2, p}}{3}\left[s^{+}\right]^{3},
$$

(ii) Let

with $C_{1, p}:=1+2^{p-3} p(p-1)$ and $C_{2, p}:=p(p-1) 2^{p-3} c_{p}{ }^{p-2}$.

$$
H(x, s):=\frac{1}{2} g(x, s) s-G(x, s) \quad \text { for } x \in \mathbb{R}_{+}^{N}, s \in \mathbb{R} .
$$

Then we have

$$
\begin{aligned}
H(x, s) \geq & \max \left\{0, \frac{p-1}{2(p+1)}\left[s^{+}\right]^{p+1}-u_{c}(x)^{p-1} D_{1, p}\left[s^{+}\right]^{2}\right. \\
& \left.-u_{c}(x) 1_{\{p>2\}} D_{2, p}\left[s^{+}\right]^{p}\right\}
\end{aligned}
$$

with $D_{1, p}:=\frac{p(p-1)}{p+1}\left(1+2^{p-2}\right)$ and $D_{2, p}:=\frac{p 2^{p-2}}{p+1}$.

Moreover, the function $H(x, \cdot)$ is non-decreasing in s for every $x \in \mathbb{R}_{+}^{N}$.

Remark 2.2 The constants $C_{i, p}$ and $D_{i, p}, i=1,2$, are not optimal. However, this choice simplifies the presentation. Moreover, they do not play an important role in our proofs below.

Proof of Lemma 2.2 (i) Since $g(\cdot, s) \equiv 0$ and $G(\cdot, s) \equiv 0$ for $s \leq 0$, it suffices to consider $s>0$. Fix $x \in \mathbb{R}_{+}^{N}$. Since $g(x, \cdot)$ is of class $C^{1}$ on $(0, \infty)$, we have 


$$
\begin{aligned}
& g(x, s)=\left(u_{c}(x)+s\right)^{p}-u_{c}(x)^{p} \\
& \quad=s p u_{c}(x)^{p-1}+p \int_{0}^{s}\left[\left(u_{c}(x)+\tau\right)^{p-1}-u_{c}(x)^{p-1}\right] d \tau, \quad \text { for } s>0 .
\end{aligned}
$$

We now distinguish two cases. If $p \in(1,2]$, we have

$$
0 \leq(a+\tau)^{p-1}-a^{p-1} \leq \tau^{p-1} \quad \text { for } \tau>0, a \geq 0,
$$

and therefore, if $p \in(1,2]$,

$$
0 \leq g(x, s)-s p u_{c}(x)^{p-1} \leq p \int_{0}^{s} \tau^{p-1} d \tau \leq s^{p}, \quad \text { for } s \geq 0 .
$$

If $p>2$, we have

$$
0 \leq(a+\tau)^{p-1}-a^{p-1} \leq(p-1) \tau(a+\tau)^{p-2}, \quad \text { for } \tau>0, a \geq 0,
$$

by the convexity of the function $a \mapsto(a+\tau)^{p-1}$ and therefore, using also (2.1),

$$
\begin{aligned}
0 & \leq g(x, s)-s p u_{c}(x)^{p-1} \leq p \int_{0}^{s}\left[\left(u_{c}(x)+\tau\right)^{p-1}-u_{c}(x)^{p-1}\right] d \tau \\
& \leq p(p-1) \int_{0}^{s} \tau\left(u_{c}(x)+\tau\right)^{p-2} d \tau \leq \frac{p(p-1)}{2} s^{2}\left(u_{c}(x)+s\right)^{p-2} \\
& \leq \frac{p(p-1)}{2} s^{2}\left(c_{p}+s\right)^{p-2} .
\end{aligned}
$$

Note also that, since $p>2$,

$$
\left(c_{p}+s\right)^{p-2} \leq\left(2 \max \left\{c_{p}, s\right\}\right)^{p-2} \leq\left(2 c_{p}\right)^{p-2}+(2 s)^{p-2}, \quad \text { for } s \geq 0 .
$$

Consequently, if $p>2$,

$0 \leq g(x, s)-s p u_{c}(x)^{p-1} \leq 2^{p-3} p(p-1) s^{p}+p(p-1) 2^{p-3} c_{p}^{p-2} s^{2}, \quad$ for $s \geq 0$.

Now (2.4) follows by combining (2.8) and (2.10). Moreover, (2.5) follows by integrating (2.4).

(ii) We first note that $H(x, s) \equiv 0$ for all $s \leq 0$. Thus, we just have to prove the result for $s>0$. Directly observe that, for all $x \in \mathbb{R}_{+}^{N}$, we have $H(x, \cdot) \in C^{1}(\mathbb{R})$ and

$$
\begin{aligned}
\frac{\partial}{\partial s} H(x, s) & =\frac{p}{2}\left(u_{c}(x)+s\right)^{p-1} s+\frac{1}{2}\left(u_{c}(x)+s\right)^{p}-\frac{1}{2} u_{c}^{p}(x)-\left(u_{c}(x)+s\right)^{p}+u_{c}^{p}(x) \\
& =\frac{1}{2}\left[p\left(u_{c}(x)+s\right)^{p-1} s-\left(u_{c}(x)+s\right)^{p}+u_{c}^{p}(x)\right] \quad \text { for }(x, s) \in \mathbb{R}_{+}^{N} \times(0,+\infty) .
\end{aligned}
$$

On the other hand, since $p>1$, we have, by the mean value theorem,

$$
\left(u_{c}(x)+s\right)^{p}-u_{c}^{p}(x) \leq p\left(u_{c}(x)+s\right)^{p-1} s \quad \text { for }(x, s) \in \mathbb{R}_{+}^{N} \times(0,+\infty) .
$$


Hence $\frac{\partial}{\partial s} H(x, s) \geq 0$ for all $s>0$, so the function $H(x, \cdot)$ is non-decrasing in $s \in[0, \infty)$. This also implies that $H(x, s) \geq 0$ for $s \geq 0$. It thus remains to prove (2.6) for $s \geq 0$. For this we first note that

$$
\begin{aligned}
H(x, s)=\frac{g(x, s) s}{2}-G(x, s)= & \frac{1}{2}\left(\left(u_{c}(x)+s\right)^{p}-u_{c}(x)^{p}\right) s \\
& -\frac{1}{p+1}\left(\left(u_{c}(x)+s\right)^{p+1}-u_{c}(x)^{p+1}-(p+1) u_{c}(x)^{p} s\right) \\
= & \left(\frac{1}{2}-\frac{1}{p+1}\right)\left(\left(u_{c}(x)+s\right)^{p}-u_{c}(x)^{p}\right) s \\
& -\frac{1}{p+1}\left(\left(u_{c}(x)+s\right)^{p}-u_{c}(x)^{p}-p u_{c}(x)^{p-1} s\right) u_{c}(x) \\
\geq & \frac{p-1}{2(p+1)} s^{p+1}-\frac{1}{p+1} u_{c}(x)\left(g(x, s)-s p u_{c}(x)^{p-1}\right)
\end{aligned}
$$

It therefore remains to show that

$$
\begin{aligned}
u_{c}(x)\left(g(x, s)-s p u_{c}^{p-1}(x)\right) \leq & (p+1)\left(u_{c}^{p-1}(x) D_{1, p} s^{2}\right. \\
& \left.+u_{c}(x) 1_{\{p>2\}} D_{2, p} s^{p}\right), \quad \text { for } s>0 .
\end{aligned}
$$

By (2.7) and integration by parts we have

$$
\begin{aligned}
u_{c}(x)\left(g(x, s)-s p u_{c}^{p-1}(x)\right) & =p u_{c}(x) \int_{0}^{s}\left[\left(u_{c}(x)+\tau\right)^{p-1}-u_{c}(x)^{p-1}\right] d \tau \\
& =p(p-1) u_{c}(x) \int_{0}^{s}(s-\tau)\left[\left(u_{c}(x)+\tau\right)^{p-2}\right] d \tau
\end{aligned}
$$

If $p \in(1,2]$, we have $\left(u_{c}(x)+\tau\right)^{p-2} \leq u_{c}(x)^{p-2}$ for $\tau>0$ and therefore

$$
\begin{aligned}
& u_{c}(x)\left(g(x, s)-s p u_{c}(x)^{p-1}\right) \\
& \leq p(p-1) u_{c}^{p-1}(x) \int_{0}^{s}(s-\tau) d \tau \leq p(p-1) u_{c}^{p-1}(x) s^{2} .
\end{aligned}
$$

If $p>2$, arguing as (2.9), we have

$$
\left(u_{c}(x)+\tau\right)^{p-2} \leq 2^{p-2}\left(u_{c}^{p-2}(x)+\tau^{p-2}\right), \quad \text { for } \tau \geq 0,
$$

and therefore (2.12) yields 


$$
\begin{gathered}
u_{c}(x)\left(g(x, s)-s p u_{c}(x)^{p-1}\right) \leq 2^{p-2} p(p-1) u_{c}(x) \int_{0}^{s}(s-\tau)\left[u_{c}^{p-2}(x)+\tau^{p-2}\right] d \tau \\
=2^{p-2} p(p-1) u_{c}(x)\left(u_{c}^{p-2}(x) \int_{0}^{s}(s-\tau) d \tau+\int_{0}^{s}(s-\tau) \tau^{p-2} d \tau\right) \\
\leq 2^{p-2} p(p-1) u_{c}^{p-1}(x) s^{2}+2^{p-2} p u_{c}(x) s^{p}, \quad \text { for } s>0 .
\end{gathered}
$$

Now (2.11) follows by combining (2.13) and (2.14). The proof is finished.

Remark 2.3 (a) From the growth estimates given in Lemma 2.2 (i) and the fact that $g$ is continuous, it follows in a standard way that the functional $E$ is well-defined on $H_{0}^{1}\left(\mathbb{R}_{+}^{N}\right)$ and of class $C^{1}$.

(b) Part (ii) of Lemma 2.2 will be useful in the analysis of Cerami sequences of the functional $E$, see Sect. 3 below.

Next, we consider the quadratic form $q_{c}: H_{0}^{1}\left(\mathbb{R}_{+}^{N}\right) \rightarrow \mathbb{R}$ given by

$$
q_{c}(u):=\int_{\mathbb{R}_{+}^{N}}\left(|\nabla u|^{2}+V_{c}(x) u^{2}\right) d x,
$$

with

$$
V_{c}(x):=1-p u_{c}^{p-1}\left(x_{N}\right) \in L^{\infty}\left(\mathbb{R}_{+}^{N}\right) .
$$

As we show in the following lemma, $q_{c}$ is positive definite on $H_{0}^{1}\left(\mathbb{R}_{+}^{N}\right)$.

Proposition 2.3 We have

$$
\widetilde{q}_{c}:=\inf _{u \in H_{0}^{1}\left(\mathbb{R}_{+}^{N}\right) \backslash\{0\}} \frac{q_{c}(u)}{\|u\|^{2}}>0 .
$$

Remark 2.4 (a) Recall that we are using the shortened notation $\|u\|^{2}=\int_{\mathbb{R}_{+}^{N}}\left(|\nabla u|^{2}+\right.$ $\left.|u|^{2}\right) d x$.

(b) From Proposition 2.3 it follows that $\left(q_{c}(\cdot)\right)^{1 / 2}$ is an equivalent norm to $\|\cdot\|$ in $H_{0}^{1}\left(\mathbb{R}_{+}^{N}\right)$.

Proof of Lemma 2.2 Since $V_{c} \in L^{\infty}\left(\mathbb{R}_{+}^{N}\right)$, it suffices to show there exists $C>0$ such that

$$
q_{c}(u) \geq C\|u\|_{L^{2}\left(\mathbb{R}_{+}^{N}\right)}^{2} \quad \text { for all } u \in H_{0}^{1}\left(\mathbb{R}_{+}^{N}\right) .
$$

Indeed, if (2.18) holds, then for $\delta \in(0,1)$ we have

$$
\begin{aligned}
q_{c}(u) \geq & \delta q_{c}(u)+(1-\delta) C\|u\|_{L^{2}\left(\mathbb{R}_{+}^{N}\right)}^{2} \geq \delta\|u\|^{2}+[(1-\delta) C \\
& \left.-\delta\left(1+\left\|V_{c}\right\|_{L^{\infty}\left(\mathbb{R}_{+}^{N}\right)}\right)\right]\|u\|_{L^{2}\left(\mathbb{R}_{+}^{N}\right)}^{2}
\end{aligned}
$$

Choosing $\delta$ sufficiently small, we have $(1-\delta) C-\delta\left(1+\left\|V_{c}\right\|_{L^{\infty}\left(\mathbb{R}_{+}^{N}\right)}\right) \geq 0$ and therefore (2.17) holds with $\widetilde{q}_{c} \geq \delta$. 
To show (2.18), we first consider the case $N=1$. Arguing by contradiction, we assume that

$$
\lambda:=\inf \left\{q_{c}(u): u \in H_{0}^{1}\left(\mathbb{R}_{+}\right) \text {and }\|u\|_{L^{2}\left(\mathbb{R}_{+}\right)}=1\right\} \leq 0
$$

(note that $\lambda>-\infty$ since $V_{c} \in L^{\infty}\left(\mathbb{R}_{+}\right)$). Then, there exists a sequence $\left(u_{n}\right)_{n}$ such that $\left\|u_{n}\right\|_{L^{2}\left(\mathbb{R}_{+}\right)}=1$ for all $n \in \mathbb{N}$ and $q_{c}\left(u_{n}\right) \rightarrow \lambda$ as $n \rightarrow \infty$. Hence, $\left(u_{n}\right)_{n}$ is a bounded sequence in $H_{0}^{1}\left(\mathbb{R}_{+}\right)$, and thus $u_{n} \rightarrow u_{*}$ weakly in $H_{0}^{1}\left(\mathbb{R}_{+}\right)$after passing to a subsequence. Moreover, with $v_{n}:=u_{n}-u_{*}$, we have $v_{n} \rightarrow 0$ in $H_{0}^{1}\left(\mathbb{R}_{+}\right)$and therefore $v_{n} \rightarrow 0$ in $L_{\text {loc }}^{2}\left(\mathbb{R}_{+}\right)$. Since $V_{c}(t) \rightarrow 1$ as $t \rightarrow \infty$, this implies that

$$
q_{c}\left(v_{n}\right) \geq \int_{\mathbb{R}_{+}} V_{c}(t) v_{n}^{2} d t \geq\left\|v_{n}\right\|_{L^{2}\left(\mathbb{R}_{+}\right)}^{2}+o(1), \quad \text { as } n \rightarrow \infty,
$$

and therefore

$$
\begin{aligned}
\lambda+o(1)= & q_{c}\left(u_{n}\right)=q_{c}\left(u_{*}\right)+q_{c}\left(v_{n}\right)+o(1) \\
\geq & \lambda\left\|u_{*}\right\|_{L^{2}\left(\mathbb{R}_{+}\right)}^{2}+\left\|v_{n}\right\|_{L^{2}\left(\mathbb{R}_{+}\right)}^{2}+o(1) \\
= & \lambda\left(\left\|u_{*}\right\|_{L^{2}\left(\mathbb{R}_{+}\right)}^{2}+\left\|v_{n}\right\|_{L^{2}\left(\mathbb{R}_{+}\right)}^{2}\right) \\
& +(1-\lambda)\left\|v_{n}\right\|_{L^{2}\left(\mathbb{R}_{+}\right)}^{2}+o(1) \\
= & \lambda+(1-\lambda)\left\|v_{n}\right\|_{L^{2}\left(\mathbb{R}_{+}\right)}^{2}+o(1)
\end{aligned}
$$

It thus follows that $v_{n} \rightarrow 0$ in $L^{2}\left(\mathbb{R}_{+}\right)$and hence $u_{n} \rightarrow u_{*}$ in $L^{2}\left(\mathbb{R}_{+}\right)$, which yields that $\left\|u_{*}\right\|_{L^{2}}=1$. Moreover, by weak lower semicontinuity of $q_{c}$ and the definition of $\lambda$, it follows that $q_{c}\left(u_{*}\right)=\lambda$, so $u_{*}$ is a constrained minimizer for $q_{c}$. A standard argument (based on replacing $u_{*}$ by $\left|u_{*}\right|$ ) shows that $u_{*} \in H_{0}^{1}\left(\mathbb{R}_{+}\right)$is a positive or negative solution of

$$
-u_{*}^{\prime \prime}+V_{c}(t) u_{*}=\lambda u_{*} \quad \text { in } \mathbb{R}_{+}, \quad u_{*}(0)=0 .
$$

Without loss of generality, we may assume that $u_{*}$ is positive, which implies that $u_{*}^{\prime}(0)>0$. We also recall that $w_{*}:=-u_{c}^{\prime}$ satisfies

$$
-w_{*}^{\prime \prime}+V_{c}(t) w_{*}=0 \quad \text { in } \mathbb{R}_{+}, \quad w_{*}>0 \quad \text { in } \overline{\mathbb{R}_{+}} .
$$

Consequently, we have

$$
0 \leq-\lambda \int_{\mathbb{R}^{+}} w_{*} u_{*} d x=\int_{\mathbb{R}^{+}}\left(w_{*} u_{*}^{\prime \prime}-u_{*} w_{*}^{\prime \prime}\right) d x=-w_{*}(0) u_{*}^{\prime}(0)<0,
$$

a contradiction. Hence, we conclude that (2.18) holds in the case $N=1$. To show (2.18) for general $N \geq 2$, we remark that, by density, we only have to show it for 
$u \in C_{c}^{\infty}\left(\mathbb{R}_{+}^{N}\right)$. For any such function we then have, writing $x=\left(x^{\prime}, t\right) \in \mathbb{R}_{+}^{N}$ with $x^{\prime} \in \mathbb{R}^{N-1}, t>0$ :

$$
\begin{aligned}
q_{c}(u) & \geq \int_{\mathbb{R}_{+}^{N}}\left(\left|\partial_{t} u\right|^{2}+V_{c} u^{2}\right) d x \\
& =\int_{\mathbb{R}^{N-1}} \int_{\mathbb{R}_{+}}\left(\left|\partial_{t} u\left(x^{\prime}, t\right)\right|^{2}+V_{c} u^{2}\left(x^{\prime}, t\right)\right) d t d x^{\prime} \\
& \geq C \int_{\mathbb{R}^{N-1}} \int_{\mathbb{R}_{+}} u^{2}\left(x^{\prime}, t\right) d t d x^{\prime}=C\|u\|_{L^{2}\left(\mathbb{R}_{+}^{N}\right)}^{2} .
\end{aligned}
$$

Here we have used the result in the case $N=1$ and the fact that $u\left(x^{\prime}, \cdot\right) \in C_{c}^{\infty}\left(\mathbb{R}_{+}\right) \subset$ $H_{0}^{1}\left(\mathbb{R}_{+}\right)$for every $x^{\prime} \in \mathbb{R}^{N-1}$. We thus have proved (2.18) for general $N \geq 1$, and the proof is complete.

Having at hand Proposition 2.3, we prove a lower estimate on the functional $E$ given in (1.15) that will be useful at several points below.

Corollary 2.4 We have

$$
E(u) \geq \frac{\tilde{q}_{c}}{2}\|u\|^{2}-\frac{C_{1, p}}{p+1}\|u\|_{L^{p+1}\left(\mathbb{R}_{+}^{N}\right)}^{p+1}-1_{\{p>2\}} \frac{C_{2, p}}{3}\|u\|_{L^{3}\left(\mathbb{R}_{+}^{N}\right)}^{3} \quad \text { for all } u \in H_{0}^{1}\left(\mathbb{R}_{+}^{N}\right)
$$

with $\widetilde{q}_{c}$ given in (2.17) and $C_{1, p}, C_{2, p}$ given in Lemma 2.2 (i).

Proof For all $u \in H_{0}^{1}\left(\mathbb{R}_{+}^{N}\right)$, we have, by (2.5) and Proposition 2.3,

$$
\begin{aligned}
E(u) & =\frac{1}{2}\|u\|^{2}-\int_{\mathbb{R}_{+}^{N}} G(x, u) d x \\
& \geq \frac{1}{2}\|u\|^{2}-\frac{1}{2} \int_{\mathbb{R}_{+}^{N}} p u_{c}^{p-1}\left(u^{+}\right)^{2} d x-\frac{C_{1, p}}{p+1}\left\|u^{+}\right\|_{L^{p+1}\left(\mathbb{R}_{+}^{N}\right)}^{p+1}-1_{\{p>2\}} \frac{C_{2, p}}{3}\left\|u^{+}\right\|_{L^{3}\left(\mathbb{R}_{+}^{N}\right)}^{3} \\
& \geq \frac{1}{2} q_{c}(u)-\frac{C_{1, p}}{p+1}\|u\|_{L^{p+1}\left(\mathbb{R}_{+}^{N}\right)}^{p+1}-1_{\{p>2\}} \frac{C_{2, p}}{3}\|u\|_{L^{3}\left(\mathbb{R}_{+}^{N}\right)}^{3} \\
& \geq \frac{\widetilde{q}_{c}}{2}\|u\|^{2}-\frac{C_{1, p}}{p+1}\|u\|_{L^{p+1}\left(\mathbb{R}_{+}^{N}\right)}^{p+1}-1_{\{p>2\}} \frac{C_{2, p}}{3}\|u\|_{L^{3}\left(\mathbb{R}_{+}^{N}\right)}^{3} .
\end{aligned}
$$

\section{Mountain-pass geometry and boundedness of the Cerami sequences}

This section is devoted to show that the functional $E$ has a Mountain-pass geometry and that, for any $d \in \mathbb{R}$, the Cerami sequences for $E$ and level $d$ are bounded. We keep using the notation of the introduction and of Sect. 2, which depends on the fixed quantities $c \in\left(0, c_{p}\right)$ and $p \in\left(1,2^{*}-1\right)$. We begin by proving that the functional $E$ has indeed a Mountain-pass geometry. 
Lemma 3.1 The functional $E$ has the following properties.

(i) $E(0)=0$.

(ii) There exist $\rho_{0}>0$ and $\delta_{0}>0$ such that $E(u) \geq \delta_{0}$ for all $u \in H_{0}^{1}\left(\mathbb{R}_{+}^{N}\right)$ such that $\|u\|=\rho_{0}$.

(iii) There exists $\psi \in H_{0}^{1}\left(\mathbb{R}_{+}^{N}\right)$ such that $\|\psi\|>\rho_{0}$ and $E(\psi)<0$.

Proof Since (i) is obvious, we concentrate on proving (ii) and (iii). We first prove (ii). Let $u \in H_{0}^{1}\left(\mathbb{R}_{+}^{N}\right)$ with $\|u\|=\rho_{0}$. By Corollary 2.4, we have

$$
E(u) \geq \frac{\tilde{q}_{c}}{2} \rho_{0}^{2}-\frac{C_{1, p}}{p+1}\|u\|_{L^{p+1}\left(\mathbb{R}_{+}^{N}\right)}^{p+1}-1_{\{p>2\}} \frac{C_{2, p}}{3}\|u\|_{L^{3}\left(\mathbb{R}_{+}^{N}\right)}^{3} .
$$

Applying then Sobolev embeddings, we deduce that

$$
E(u) \geq \frac{\widetilde{q}_{c}}{2} \rho_{0}^{2}-C\left(\rho_{0}^{p+1}+1_{\{p>2\}} \rho_{0}^{3}\right),
$$

with a constant $C>0$. Since $p>1$, Claim (ii) follows by taking $\rho_{0}$ sufficiently small. It then remains to prove (iii). Let $\varphi \in C_{c}^{\infty}\left(\mathbb{R}_{+}^{N}\right)$ with $\varphi \supsetneqq 0$ and $\psi:=t \varphi$ with $t \in(0,+\infty)$. Directly observe that

$$
E(\psi)=\frac{t^{2}}{2}\|\varphi\|^{2}+t \int_{\mathbb{R}_{+}^{N}} u_{c}^{p} \varphi d x-\frac{1}{p+1} \int_{\mathbb{R}_{+}^{N}}\left(\left(u_{c}+t \varphi\right)^{p+1}-u_{c}^{p+1}\right) d x
$$

Then, since

$$
\int_{\mathbb{R}_{+}^{N}}\left(\left(u_{c}+t \varphi\right)^{p+1}-u_{c}^{p+1}\right) d x \geq t^{p+1}\|\varphi\|_{L^{p+1}\left(\mathbb{R}_{+}^{N}\right)}^{p+1},
$$

we have that

$$
E(\psi) \leq \frac{t^{2}}{2}\|\varphi\|^{2}+t c^{p}\|\varphi\|_{L^{1}\left(\mathbb{R}_{+}^{N}\right)}-\frac{t^{p+1}}{p+1}\|\varphi\|_{L^{p+1}\left(\mathbb{R}_{+}^{N}\right)}^{p+1} .
$$

Claim (iii) follows taking $t$ sufficiently large and thus the proof is complete.

We now prove the boundedness of Cerami sequences of the functional $E$.

Proposition 3.2 Cerami sequences for $E$ at any level $d \in \mathbb{R}$ are bounded.

Remark 3.1 (a) Recall that $\left(\varphi_{n}\right)_{n} \subset H_{0}^{1}\left(\mathbb{R}_{+}^{N}\right)$ is a Cerami sequence for $E$ at level $d \in \mathbb{R}$ if

$$
E\left(\varphi_{n}\right) \rightarrow d \quad \text { and } \quad\left(1+\left\|\varphi_{n}\right\|\right)\left\|E^{\prime}\left(\varphi_{n}\right)\right\|_{H^{-1}\left(\mathbb{R}_{+}^{N}\right)} \rightarrow 0
$$

(b) The proof of Proposition 3.2 is inspired by [21, Sect. 3]. However, since our problem is not invariant under translations in $\mathbb{R}^{N}$ and our nonlinearity $g$ has a non-standard shape, several difficulties appear. 
Proof of Proposition 3.2 Let $d \in \mathbb{R}$ be an arbitrary but fixed constant and let $\left(u_{n}\right)_{n} \subset$ $H_{0}^{1}\left(\mathbb{R}_{+}^{N}\right)$ be a Cerami sequence for $E$ at level $d \in \mathbb{R}$. First of all, observe that

$$
\left\|u_{n}^{-}\right\|^{2}=-\left\langle E^{\prime}\left(u_{n}\right), u_{n}^{-}\right\rangle \rightarrow 0, \quad \text { as } n \rightarrow \infty .
$$

In particular, we deduce that $\left(u_{n}^{-}\right)_{n}$ is bounded. It then remains to prove that $\left(u_{n}^{+}\right)_{n}$ is bounded. We assume by contradiction that $\left\|u_{n}\right\| \rightarrow \infty$ and we set $v_{n}:=u_{n} /\left\|u_{n}\right\|$ for all $n \in \mathbb{N}$. Since $\left(v_{n}\right)_{n}$ and $\left(u_{n}^{-}\right)_{n}$ are bounded, up to a subsequence if necessary, we have

$$
v_{n} \rightarrow v \text { in } H_{0}^{1}\left(\mathbb{R}_{+}^{N}\right), \quad v_{n} \rightarrow v \text { in } L_{l o c}^{q}\left(\mathbb{R}_{+}^{N}\right) \quad \text { for } 1 \leq q<2^{*} \quad \text { and } \quad v_{n} \rightarrow v \text { a.e. in } \mathbb{R}_{+}^{N},
$$

for some $v \in H_{0}^{1}\left(\mathbb{R}_{+}^{N}\right)$ with $v \geq 0$. We have now two possible cases:

Case 1 (Vanishing): For all $R>0$, it follows that

$$
\lim _{n \rightarrow \infty} \sup _{y \in \mathbb{R}_{+}^{N}} \int_{B_{R}(y) \cap \mathbb{R}_{+}^{N}} v_{n}^{2} d x=0 .
$$

Case 2 (Non-vanishing): There exist $R>0, \delta>0$ and a sequence of points $\left(y^{n}\right)_{n} \subset$ $\mathbb{R}_{+}^{N}$ such that

$$
\lim _{n \rightarrow \infty} \int_{B_{R}\left(y^{n}\right) \cap \mathbb{R}_{+}^{N}} v_{n}^{2} d x \geq \delta .
$$

We shall prove that none of these cases may happen. This will prove the boundedness of the sequence Cerami sequence $\left(u_{n}\right)_{n}$.

Case 1 (Vanishing): First of all, observe that, by (3.2) and Lions' Lemma [25, Lemma I.1], $v_{n} \rightarrow 0$ in $L^{q}\left(\mathbb{R}_{+}^{N}\right)$ for all $2<q<2^{*}$, and so, by uniqueness of the limit we have $v \equiv 0$. We define then the sequence $\left(z_{n}\right)_{n} \subset H_{0}^{1}\left(\mathbb{R}_{+}^{N}\right)$ by $z_{n}:=t_{n} u_{n}$ with $t_{n} \in[0,1]$ satisfying

$$
E\left(z_{n}\right)=\max _{t \in[0,1]} E\left(t u_{n}\right)
$$

(if, for $n \in \mathbb{N}, t_{n}$ is not unique, we choose the smallest value) and we split the proof in the vanishing case (Case 1) into three steps.

Step 1.1: $\lim _{n \rightarrow \infty} E\left(z_{n}\right)=+\infty$.

We argue by contradiction. Suppose there exists $M<+\infty$ such that

$$
\liminf _{n \rightarrow \infty} E\left(z_{n}\right) \leq M
$$

and define $\left(k_{n}\right)_{n} \subset H_{0}^{1}\left(\mathbb{R}_{+}^{N}\right)$ as

$$
k_{n}:=\left(\frac{4 M}{\widetilde{q}_{c}}\right)^{\frac{1}{2}} v_{n}=\left(\frac{4 M}{\widetilde{q}_{c}}\right)^{\frac{1}{2}} \frac{1}{\left\|u_{n}\right\|} u_{n}, \quad \text { for all } n \in \mathbb{N},
$$


where $\widetilde{q}_{c}>0$ is the constant given by Proposition 2.3. First, observe that

$$
k_{n} \rightarrow 0 \text { in } H_{0}^{1}\left(\mathbb{R}_{+}^{N}\right), \quad k_{n} \rightarrow 0 \text { in } L^{q}\left(\mathbb{R}_{+}^{N}\right), \text { for } 2<q<2^{*}, \quad \text { and } \quad k_{n} \rightarrow 0 \text { a.e. in } \mathbb{R}_{+}^{N} \text {. }
$$

Then, by Corollary 2.4 and (3.4), we obtain that

$$
E\left(k_{n}\right) \geq \frac{\widetilde{q}_{c}}{2}\left\|k_{n}\right\|^{2}-C\left(\left\|k_{n}\right\|_{L^{p+1}\left(\mathbb{R}_{+}^{N}\right)}^{p+1}+1_{\{p>2\}}\left\|k_{n}\right\|_{L^{3}\left(\mathbb{R}_{+}^{N}\right)}^{3}\right)=2 M+o(1) .
$$

Taking $M$ bigger if necessary, we have that, for all $n \in \mathbb{N}$ large enough,

$$
E\left(k_{n}\right)>\frac{3}{2} M
$$

On the other hand, observe that, for $n \in \mathbb{N}$ large enough, $\left(\frac{4 M}{\widetilde{q}_{c}}\right)^{\frac{1}{2}} \frac{1}{\left\|u_{n}\right\|} \in[0,1]$. Hence, we have that

$$
\frac{3}{2} M \leq \liminf _{n \rightarrow \infty} E\left(k_{n}\right) \leq \liminf _{n \rightarrow \infty} E\left(z_{n}\right) \leq M
$$

which is a contradiction. Thus, the Step 1.1 follows.

Step 2.1: $\left\langle E^{\prime}\left(z_{n}\right), z_{n}\right\rangle=0$ for all $n \in \mathbb{N}$ large enough.

By Step 1.1 we know that $E\left(z_{n}\right) \rightarrow \infty$ as $n \rightarrow \infty$. On the other hand, $E(0)=0$ and $E\left(u_{n}\right) \rightarrow d$ as $n \rightarrow \infty$. Hence, for $n \in \mathbb{N}$ large enough, $t_{n} \in(0,1)$ and so, by the definition of $z_{n}$, the Step 2.1 follows.

\section{Step 3.1: Conclusion Case 1.}

Observe that, by Step 2.1, for all $n \in \mathbb{N}$ large enough,

$$
E\left(z_{n}\right)=E\left(z_{n}\right)-\frac{1}{2}\left\langle E^{\prime}\left(z_{n}\right), z_{n}\right\rangle=\int_{\mathbb{R}_{+}^{N}} H\left(x, z_{n}\right) d x
$$

where $H$ is given in Lemma 2.2 (ii). By Step 1.1, we have that

$$
\lim _{n \rightarrow \infty} \int_{\mathbb{R}_{+}^{N}} H\left(x, z_{n}\right) d x=+\infty
$$

On the other hand, since $\left(u_{n}\right)_{n}$ is a Cerami sequence,

$$
d+o(1)=E\left(u_{n}\right)-\frac{1}{2}\left\langle E^{\prime}\left(u_{n}\right), u_{n}\right\rangle=\int_{\mathbb{R}_{+}^{N}} H\left(x, u_{n}\right) d x
$$


Then, using the definition of $z_{n}$ and the fact that $H(x, s)$ is non-decreasing in $s$ by Lemma 2.2 (ii), we obtain

$$
\int_{\mathbb{R}_{+}^{N}} H\left(x, z_{n}\right) d x \leq \int_{\mathbb{R}_{+}^{N}} H\left(x, u_{n}\right) d x=d+o(1),
$$

which clearly contradicts (3.6). Hence, Case 1 (vanishing) cannot happen.

Case 2 (Non-vanishing): We split the proof into two steps.

Step 1.2: There exists $M>0$ such that $y_{N}^{n}:=\operatorname{dist}\left(y^{n}, \partial \mathbb{R}_{+}^{N}\right) \leq M$ for all $n \in \mathbb{N}$.

We assume by contradiction that $y_{N}^{n} \rightarrow+\infty$ as $n \rightarrow+\infty$. Then, for all $n \in \mathbb{N}$, we introduce $w_{n}:=v_{n}\left(\cdot+y^{n}\right)$ and observe that

$$
w_{n} \rightarrow w \text { in } H^{1}\left(\mathbb{R}^{N}\right), \quad w_{n} \rightarrow w \text { in } L_{\text {loc }}^{q}\left(\mathbb{R}^{N}\right) \text { for } 1 \leq q<2^{*}, \quad \text { and } \quad w_{n} \rightarrow w \text { a.e. in } \mathbb{R}^{N},
$$

for some $w \in H^{1}\left(\mathbb{R}^{N}\right)$ with $w \not \equiv 0$ (by (3.3)) and $w \geq 0$. Now, observe that, since $\left(u_{n}\right)_{n}$ is a Cerami sequence, Lemma 2.2 (ii) implies that

$$
\begin{aligned}
& o(1)=\frac{1}{\left\|u_{n}\right\|^{p+1}}\left(E\left(u_{n}\right)-\frac{1}{2}\left\langle E^{\prime}\left(u_{n}\right), u_{n}\right\rangle\right) \\
& =\frac{1}{\left\|u_{n}\right\|^{p+1}} \int_{\mathbb{R}_{+}^{N}} H\left(x, u_{n}(x)\right) d x \\
& \geq \frac{1}{\left\|u_{n}\right\|^{p+1}}\left[\frac{p-1}{2(p+1)}\left\|u_{n}^{+}\right\|_{L^{p+1}\left(\mathbb{R}_{+}^{N}\right)}^{p+1}\right. \\
& \left.-\int_{\mathbb{R}_{+}^{N}}\left(u_{c}^{p-1}(x) D_{1, p}\left[u_{n}^{+}\right]^{2}+u_{c}(x) 1_{\{p>2\}} D_{2, p}\left[u_{n}^{+}\right]^{p}\right) d x\right] \\
& \geq \frac{1}{\left\|u_{n}\right\|^{p+1}}\left[\frac{p-1}{2(p+1)}\left\|u_{n}^{+}\right\|_{L^{p+1}\left(\mathbb{R}_{+}^{N}\right)}^{p+1}\right. \\
& \left.-\max \left\{c_{p}{ }^{p-1}, c_{p}\right\}\left(D_{1, p}\left\|u_{n}^{+}\right\|_{L^{2}\left(\mathbb{R}_{+}^{N}\right)}^{2}+1_{\{p>2\}} D_{2, p}\left\|u_{n}^{+}\right\|_{L^{p}\left(\mathbb{R}_{+}^{N}\right)}^{p}\right)\right] \\
& \geq \frac{p-1}{2(p+1)}\left\|v_{n}^{+}\right\|_{L^{p+1}\left(\mathbb{R}_{+}^{N}\right)}^{p+1} \\
& -\frac{C}{\left\|u_{n}\right\|^{p+1}}\left(\left\|u_{n}\right\|^{2}+1_{\{p>2\}}\left\|u_{n}\right\|^{p}\right) \geq \frac{p-1}{2(p+1)}\left\|v_{n}^{+}\right\|_{L^{p+1}\left(\mathbb{R}_{+}^{N}\right)}^{p+1}+o(1),
\end{aligned}
$$

where $C>0$ is a constant independent of $n$. Here we also used Sobolev embeddings and the fact that $\left\|u_{n}\right\| \rightarrow \infty$ as $n \rightarrow \infty$. Since $p>1$, we thus conclude by Fatou's Lemma that

$$
0=\lim _{n \rightarrow \infty}\left\|v_{n}^{+}\right\|_{L^{p+1}\left(\mathbb{R}_{+}^{N}\right)}^{p+1}=\liminf _{n \rightarrow \infty} \int_{\left\{x_{N} \geq-y_{N}^{n}\right\}}\left(w_{n}^{+}\right)^{p+1} d x \geq \int_{\mathbb{R}^{N}}\left(w^{+}\right)^{p} d x
$$

Hence $w=w^{+} \equiv 0$, which clearly is a contradiction. Thus, Step 1.2 follows. 


\section{Step 2.2: Conclusion Case 2.}

By Step 1.2 we know there exists $M>0$ such that $y_{N}^{n} \leq M$ for all $n \in \mathbb{N}$. We then define, for all $n \in \mathbb{N}, \widetilde{w}_{n}:=v_{n}\left(\cdot+\xi_{n}\right)$, where $\xi_{n}=\left(y_{1}^{n}, \ldots, y_{N-1}^{n}, 0\right)$. Again by (3.3), we have

$$
\begin{aligned}
& \widetilde{w}_{n} \rightarrow \widetilde{w} \text { in } H_{0}^{1}\left(\mathbb{R}_{+}^{N}\right), \quad \widetilde{w}_{n} \rightarrow \widetilde{w} \text { in } L_{l o c}^{q}\left(\mathbb{R}_{+}^{N}\right) \text { for } 1 \leq q<2^{*}, \quad \text { and } \\
& \widetilde{w}_{n} \rightarrow \widetilde{w} \text { a.e. in } \mathbb{R}_{+}^{N},
\end{aligned}
$$

for some $\widetilde{w} \in H_{0}^{1}\left(\mathbb{R}_{+}^{N}\right)$ with $\widetilde{w} \not \equiv 0$ and $\widetilde{w} \geq 0$. For $n \in \mathbb{N}$, let $\varphi_{n}:=\widetilde{w}\left(\cdot-\xi_{n}\right) \in$ $H_{0}^{1}\left(\mathbb{R}_{+}^{N}\right)$. Since $\left(u_{n}\right)_{n}$ is a Cerami sequence with $\left\|u_{n}\right\| \rightarrow \infty$ as $n \rightarrow \infty$, we have

$$
\begin{aligned}
o(1)= & \frac{\left\langle E^{\prime}\left(u_{n}\right), \varphi_{n}\right\rangle}{\left\|u_{n}\right\|} \\
= & \frac{1}{\left\|u_{n}\right\|} \int_{\mathbb{R}_{+}^{N}}\left(\nabla u_{n} \nabla \varphi_{n}+u_{n} \varphi_{n}-g\left(x, u_{n}\right) \varphi_{n}\right) d x \\
= & \int_{\mathbb{R}_{+}^{N}}\left(\nabla v_{n} \nabla \varphi_{n}+v_{n} \varphi_{n}\right) d x \\
& -\int_{\mathbb{R}_{+}^{N}}\left(\frac{\left(u_{c}+u_{n}^{+}\right)^{p}-u_{c}^{p}}{\left\|u_{n}\right\|}\right) \varphi_{n} d x \\
= & \int_{\mathbb{R}_{+}^{N}}\left(\nabla \widetilde{w}_{n} \nabla \widetilde{w}+\widetilde{w}_{n} \widetilde{w}\right) d x \\
& -\int_{\mathbb{R}_{+}^{N}}\left(\frac{\left(u_{c}+\left\|u_{n}\right\| \widetilde{w}_{n}^{+}\right)^{p}-u_{c}^{p}}{\left\|u_{n}\right\|}\right) \tilde{w}^{p} d x \\
= & \|\widetilde{w}\|^{2}+o(1)-\int_{\mathbb{R}_{+}^{N}}\left(\frac{\left(u_{c}+\left\|u_{n}\right\| \widetilde{w}_{n}^{+}\right)^{p}-u_{c}^{p}}{\left\|u_{n}\right\|}\right) \tilde{w} d x .
\end{aligned}
$$

On the other hand, since $p>1$, we have that

$$
\liminf _{n \rightarrow \infty} \frac{\left(u_{c}+\left\|u_{n}\right\| \widetilde{w}_{n}^{+}\right)^{p}-u_{c}^{p}}{\left\|u_{n}\right\| \widetilde{w}_{n}^{+}} \widetilde{w}_{n}^{+} \widetilde{w}=+\infty, \quad \text { a.e. in }\{\widetilde{w}>0\}
$$

and therefore, since $\widetilde{w} \geq 0$ and $\widetilde{w} \not \equiv 0$,

$$
\begin{aligned}
& \liminf _{n \rightarrow \infty} \int_{\mathbb{R}_{+}^{N}}\left(\frac{\left(u_{c}+u_{n}^{+}\left(\cdot+\xi_{n}\right)\right)^{p}-u_{c}^{p}}{\left\|u_{n}\right\|}\right) \widetilde{w} d x \\
& \quad=\liminf _{n \rightarrow \infty} \int_{\{\tilde{w}>0\}}\left(\frac{\left(u_{c}+u_{n}^{+}\left(\cdot+\xi_{n}\right)\right)^{p}-u_{c}^{p}}{\left\|u_{n}\right\|}\right) \widetilde{w} d x=+\infty
\end{aligned}
$$

by Fatou's Lemma. This yields a contradiction. Hence, Case 2 (non-vanishing) cannot happen either and thus the result follows. 
Lemma 3.3 Let $\left(u_{n}\right)_{n}$ be a Cerami sequence for $E$ at level $d \in \mathbb{R} \backslash\{0\}$. Then, there exist $R>0, \delta>0$ and a sequence of points $\left(y^{n}\right)_{n} \subset \mathbb{R}_{+}^{N}$ such that

$$
\liminf _{n \rightarrow \infty} \int_{B_{R}\left(y^{n}\right) \cap \mathbb{R}_{+}^{N}} u_{n}^{2} d x \geq \delta .
$$

Proof We assume by contradiction that, for all $R>0$,

$$
\liminf _{n \rightarrow \infty} \sup _{y \in \mathbb{R}_{+}^{N}} \int_{B_{R}(y) \cap \mathbb{R}_{+}^{N}} u_{n}^{2} d x=0 .
$$

Then, by Lions' [25, Lemma I.1], we have that $u_{n} \rightarrow 0$ in $L^{q}\left(\mathbb{R}_{+}^{N}\right)$ for all $2<q<2^{*}$. Now, since $\left(u_{n}\right)_{n}$ is a Cerami sequence, using Lemma 2.2 (i), we get

$$
\begin{aligned}
o(1) & =\left\langle E^{\prime}\left(u_{n}\right), u_{n}\right\rangle=\left\|u_{n}\right\|^{2}-\int_{\mathbb{R}_{+}^{N}} g\left(x, u_{n}(x)\right) u_{n} d x \\
& \geq\left\|u_{n}\right\|^{2}-\int_{\mathbb{R}_{+}^{N}} p u_{c}^{p-1}\left(u_{n}^{+}\right)^{2} d x-C_{1, p}\left\|u_{n}^{+}\right\|_{L^{p+1}\left(\mathbb{R}_{+}^{N}\right)}^{p+1}-1_{\{p>2\}} C_{2, p}\left\|u_{n}^{+}\right\|_{L^{3}\left(\mathbb{R}_{+}^{N}\right)}^{3} \\
& \geq q_{c}\left(u_{n}\right)-C_{1, p}\left\|u_{n}\right\|_{L^{p+1}\left(\mathbb{R}_{+}^{N}\right)}^{p+1}-1_{\{p>2\}} C_{2, p}\left\|u_{n}\right\|_{L^{3}\left(\mathbb{R}_{+}^{N}\right)}^{3} \\
& \geq \widetilde{q}_{c}\left\|u_{n}\right\|^{2}-C_{1, p}\left\|u_{n}\right\|_{L^{p+1}\left(\mathbb{R}_{+}^{N}\right)}^{p+1}-1_{\{p>2\}} C_{2, p}\left\|u_{n}\right\|_{L^{3}\left(\mathbb{R}_{+}^{N}\right)}^{3} .
\end{aligned}
$$

Hence, since $u_{n} \rightarrow 0$ in $L^{q}\left(\mathbb{R}_{+}^{N}\right)$ for all $2<q<2^{*}$, we deduce that $\left\|u_{n}\right\| \rightarrow 0$. Since $E$ is continuous, this implies that $E\left(u_{n}\right) \rightarrow 0$ as $n \rightarrow \infty$, contradicting our assumption that $d \neq 0$. The proof is finished.

\section{Energy estimates}

We keep using the notation of the introduction and of Sect. 2, which depends on the fixed quantities $c \in\left(0, c_{p}\right)$ and $p \in\left(1,2^{*}-1\right)$. Moreover, we will assume $N \geq 2$ throughout this section, which will be of key importance in order to derive the energy estimates we need. The mountain pass value associated to (1.14) is given by

$$
b:=\inf _{\gamma \in \Gamma} \max _{t \in[0,1]} E(\gamma(t))
$$

where

$$
\Gamma:=\left\{\gamma \in C\left([0,1], H_{0}^{1}\left(\mathbb{R}_{+}^{N}\right)\right): \gamma(0)=0, E(\gamma(1))<0\right\}
$$

We note that $b>0$ by Lemma 3.1. We also note that the functional $E$ (given in (1.15)) can be written as 


$$
\begin{aligned}
E(u) & =E_{\infty}^{+}(u) \\
& -\frac{1}{p+1} \int_{\mathbb{R}_{+}^{N}}\left(\left(u_{c}+u^{+}\right)^{p+1}-u_{c}^{p+1}-\left(u^{+}\right)^{p+1}-(p+1) u_{c}^{p} u^{+}\right) d x,
\end{aligned}
$$

where $E_{\infty}^{+}: H_{0}^{1}\left(\mathbb{R}_{+}^{N}\right) \rightarrow \mathbb{R}$ is given by

$$
E_{\infty}^{+}(u)=\frac{1}{2}\|u\|^{2}-\frac{1}{p+1} \int_{\mathbb{R}_{+}^{N}}\left(u^{+}\right)^{p+1} d x
$$

Now, we introduce the auxiliary (limit) problem

$$
-\Delta u+u=|u|^{p-1} u, \quad u \in H^{1}\left(\mathbb{R}^{N}\right),
$$

and its associated energy $E_{\infty}: H^{1}\left(\mathbb{R}^{N}\right) \rightarrow \mathbb{R}$ given by

$$
E_{\infty}(u)=\frac{1}{2} \int_{\mathbb{R}^{N}}\left(|\nabla u|^{2}+u^{2}\right) d x-\frac{1}{p+1} \int_{\mathbb{R}^{N}}|u|^{p+1} d x .
$$

Also, we define

$$
b_{\infty}:=\inf _{\mathcal{K}_{\infty}} E_{\infty}, \quad \text { where } \quad \mathcal{K}_{\infty}:=\left\{u \in H^{1}\left(\mathbb{R}^{N}\right) \backslash\{0\}: E_{\infty}^{\prime}(u)=0\right\}
$$

According to [8, Theorem 1], [6, Théorème 1] and [16, Theorem 2], there exists a ground-state solution $\psi \in C^{2}\left(\mathbb{R}^{N}\right)$ to (4.3) which is positive, radially symmetric, and such that

$\psi(x) \leq C_{G S}|x|^{-\frac{N-1}{2}} e^{-|x|} \quad$ and $\quad|\nabla \psi(x)| \leq C_{G S}|x|^{-\frac{N-1}{2}} e^{-|x|}, \quad$ as $\quad|x| \rightarrow \infty$

for some $C_{G S}>0$ depending only on $N$ and $p$. Moreover,

$\psi$ is strictly decreasing in the radial variable.

Let us also emphasize that

$$
\begin{aligned}
0<b_{\infty} & =E_{\infty}(\psi)=\left(\frac{1}{2}-\frac{1}{p+1}\right)\|\psi\|_{L^{p+1}\left(\mathbb{R}^{N}\right)}^{p+1}=\frac{p-1}{2(p+1)}\|\psi\|_{L^{p+1}\left(\mathbb{R}^{N}\right)}^{p+1} \\
& =\frac{p-1}{2(p+1)} \inf _{u \in \mathcal{K}_{\infty}}\|u\|_{L^{p+1}\left(\mathbb{R}^{N}\right)}^{p+1}
\end{aligned}
$$

The aim of this section is to show, based on the assumption $N \geq 2$, that

$$
b<b_{\infty}
$$


This strict inequality will be crucial to prove the existence result to (1.14) contained in Sect. 5. To this end, let us recall that $u_{c}(x) \sim e^{-x_{N}}$ as $x_{N} \rightarrow \infty$. More precisely, it follows from (1.6) and the definition of $u_{c}$ that

$$
\begin{aligned}
m_{c, 1} e^{-x_{N}} & \leq u_{c}(x) \leq m_{c, 2} e^{-x_{N}}, \quad \text { for } x \in \mathbb{R}_{+}^{N}, \quad \text { with } \\
m_{c, 1} & :=c_{p} e^{-t_{c, p}}, \quad m_{c, 2}:=c_{p} 2^{\frac{2}{p-1}} e^{-t_{c, p}}
\end{aligned}
$$

Moreover, for $r>0$, we introduce the function

$$
x \mapsto \psi_{r}(x):=\left(\psi\left(x-r e_{N}\right)-\varepsilon_{r}\right)^{+},
$$

where $e_{N}=(0, \ldots, 0,1)$ is the $N$-th coordinate vector and $\varepsilon_{r}>0$ is uniquely defined by (4.7) and the property that

$$
\psi>\varepsilon_{r} \quad \text { in } B_{r}(0) \quad \text { and } \quad \psi \leq \varepsilon_{r} \quad \text { in } \mathbb{R}^{N} \backslash B_{r}(0) .
$$

We note that, as a consequence of (4.6), we have

$$
\varepsilon_{r} \leq C_{G S} r^{-\frac{N-1}{2}} e^{-r}
$$

We also note that $\psi_{r} \in H_{0}^{1}\left(\mathbb{R}_{+}^{N}\right)$ for every $r \geq 0$.

The rest of the section is devoted to prove the following result from which (4.9) immediately follows.

Proposition 4.1 There exists $R>0$ and $k>0$ with the following properties:

(i) $E\left(t \psi_{r}\right)<b_{\infty}$ for all $r \geq R, t \in[0, k]$.

(ii) $E\left(k \psi_{r}\right)<0$ for all $r \geq R$.

We split the proof of this proposition into several lemmas.

Lemma 4.2 There exists $C_{1}>0$ with

$$
E_{\infty}^{+}\left(t \psi_{r}\right) \leq b_{\infty}+C_{1} e^{-r} r^{-\frac{N-1}{2}} t^{p+1} \quad \text { for all } t, r>0
$$

Proof Let $t, r>0$. Directly observe that, by the definition of $\psi_{r}$,

$$
\begin{aligned}
E_{\infty}^{+}\left(t \psi_{r}\right) & =\frac{t^{2}}{2}\left\|\psi_{r}\right\|^{2}-\frac{t^{p+1}}{p+1} \int_{\mathbb{R}_{+}^{N}} \psi_{r}^{p+1} d x \\
& =\frac{t^{2}}{2} \int_{B_{r}(0)}\left(|\nabla \psi|^{2}+\left(\psi-\varepsilon_{r}\right)^{2}\right) d x-\frac{t^{p+1}}{p+1} \int_{B_{r}(0)}\left(\psi-\varepsilon_{r}\right)^{p+1} d x
\end{aligned}
$$


On the other hand, since $\psi$ is a solution to (4.3) and $\left(\psi-\varepsilon_{r}\right)^{+} \in H^{1}\left(\mathbb{R}^{N}\right)$, using $\left(\psi-\varepsilon_{r}\right)^{+}$as test function in (4.3), we obtain that

$$
\begin{aligned}
\int_{B_{r}(0)}|\nabla \psi|^{2} d x & =\int_{\mathbb{R}^{N}} \nabla \psi \nabla\left(\psi-\varepsilon_{r}\right)^{+} d x \\
& =\int_{\mathbb{R}^{N}}\left(-\psi\left(\psi-\varepsilon_{r}\right)^{+}+\psi^{p}\left(\psi-\varepsilon_{r}\right)^{+}\right) d x \\
& =\int_{B_{r}(0)}\left(-\psi\left(\psi-\varepsilon_{r}\right)+\psi^{p}\left(\psi-\varepsilon_{r}\right)\right) d x
\end{aligned}
$$

Substituting the above identity into (4.13) and using the mean value theorem, we find that

$$
\begin{aligned}
& E_{\infty}^{+}\left(t \psi_{r}\right)=\frac{t^{2}}{2} \int_{B_{r}(0)}\left(-\psi\left(\psi-\varepsilon_{r}\right)+\psi^{p}\left(\psi-\varepsilon_{r}\right)+\left(\psi-\varepsilon_{r}\right)^{2}\right) d x \\
& -\frac{t^{p+1}}{p+1} \int_{B_{r}(0)}\left(\psi-\varepsilon_{r}\right)^{p+1} d x \\
& =-\frac{\varepsilon_{r} t^{2}}{2} \int_{B_{r}(0)}\left(\psi-\varepsilon_{r}\right) d x \\
& +\frac{1}{p+1} \int_{B_{r}(0)}\left(\psi-\varepsilon_{r}\right)\left[\frac{p+1}{2} t^{2} \psi^{p}-t^{p+1}\left(\psi-\varepsilon_{r}\right)^{p}\right] d x \\
& \leq \frac{1}{p+1} \int_{B_{r}(0)}\left(\psi-\varepsilon_{r}\right)\left[\frac{p+1}{2} t^{2} \psi^{p}-t^{p+1}\left(\psi-\varepsilon_{r}\right)^{p}\right] d x \\
& =\frac{1}{p+1}\left(\frac{p+1}{2} t^{2}-t^{p+1}\right) \int_{B_{r}(0)}\left(\psi-\varepsilon_{r}\right) \psi^{p} d x \\
& +\frac{t^{p+1}}{p+1} \int_{B_{r}(0)}\left(\psi-\varepsilon_{r}\right)\left[\psi^{p}-\left(\psi-\varepsilon_{r}\right)^{p}\right] d x \\
& \leq \frac{p-1}{2(p+1)} \int_{B_{r}(0)}\left(\psi-\varepsilon_{r}\right) \psi^{p} d x+\frac{\varepsilon_{r} p t^{p+1}}{p+1} \int_{B_{r}(0)}\left(\psi-\varepsilon_{r}\right) \psi^{p-1} d x \\
& \leq \frac{p-1}{2(p+1)} \int_{\mathbb{R}^{N}} \psi^{p+1} d x+\frac{\varepsilon_{r} p t^{p+1}}{p+1} \int_{\mathbb{R}^{N}}\left(\psi-\varepsilon_{r}\right)^{+} \psi^{p-1} d x .
\end{aligned}
$$

Using (4.8) and (4.11), we deduce that

$$
\begin{aligned}
E_{\infty}^{+}\left(t \psi_{r}\right) \leq & b_{\infty}+\frac{\varepsilon_{r} p t^{p+1}}{p+1} \int_{\mathbb{R}^{N}}\left(\psi-\varepsilon_{r}\right)^{+} \psi^{p-1} d x \leq b_{\infty} \\
& +\frac{p}{p+1} C_{G S} r^{-\frac{N-1}{2}} e^{-r} t^{p+1} \int_{\mathbb{R}^{N}} \psi^{p} d x
\end{aligned}
$$

Hence (4.12) holds with $C_{1}=\frac{p C_{G S}}{p+1} \int_{\mathbb{R}^{N}} \psi^{p} d x$. 
Lemma 4.3 There exists $R^{\prime} \geq 1$ and $C_{2}>0$ with

$$
E_{\infty}^{+}\left(t \psi_{r}\right)-E\left(t \psi_{r}\right) \geq C_{2} e^{-r} t^{p} \quad \text { for all } t>0, r \geq R^{\prime}
$$

Proof Let $t>0$ be arbitrary but fixed. Using Lemma 2.1 with $q=p+1, \kappa:=\kappa_{q}>0$, the identity (4.2), the lower bound in (4.10) and the mean value theorem, we deduce that, for all $r \geq 1$,

$$
\begin{aligned}
E_{\infty}^{+}\left(t \psi_{r}\right)-E\left(t \psi_{r}\right)= & \frac{1}{p+1} \int_{\mathbb{R}_{+}^{N}}\left(\left(u_{c}+t \psi_{r}\right)^{p+1}-u_{c}^{p+1}\right. \\
& \left.-\left(t \psi_{r}\right)^{p+1}-(p+1) u_{c}^{p} t \psi_{r}\right) d x \geq \kappa t^{p} \int_{\mathbb{R}_{+}^{N}} u_{c} \psi_{r}^{p} d x \\
= & \kappa t^{p} \int_{\mathbb{R}_{+}^{N}} u_{c}\left(\left(\psi\left(\cdot-r e_{N}\right)-\varepsilon_{r}\right)^{+}\right)^{p} d x \\
= & \kappa t^{p} \int_{B_{r}(0)} u_{c}\left(\cdot+r e_{N}\right)\left(\psi-\varepsilon_{r}\right)^{p} d x \\
\geq & \kappa t^{p} \int_{B_{r}(0)} u_{c}\left(\cdot+r e_{N}\right)\left(\psi^{p}-\varepsilon_{r} p \psi^{p-1}\right) d x \\
\geq & \kappa t^{p}\left(\int_{B_{r}^{-}(0)} u_{c}\left(\cdot+r e_{N}\right) \psi^{p} d x-\varepsilon_{r} p c_{p} \int_{\mathbb{R}^{N}} \psi^{p-1} d x\right) \\
\geq & \kappa t^{p}\left(m_{c, 1} e^{-r} \int_{B_{r}^{-}(0)} \psi^{p} d x\right. \\
& \left.-p c_{p} C_{G S} r^{-\frac{N-1}{2}} e^{-r} \int_{\mathbb{R}^{N}} \psi^{p-1} d x\right) \\
\geq & \kappa t^{p} e^{-r}\left(\frac{m_{c, 1}}{2} \int_{B_{r}(0)} \psi^{p} d x-p c_{p} C_{G S} r^{-\frac{N-1}{2}} \int_{\mathbb{R}^{N}} \psi^{p-1} d x\right) \\
\geq & \kappa t^{p} e^{-r}\left(\frac{m_{c, 1}}{2} \int_{B_{1}(0)} \psi^{p} d x-p c_{p} C_{G S} r^{-\frac{N-1}{2}} \int_{\mathbb{R}^{N}} \psi^{p-1} d x\right) \\
& \\
& \\
& \\
&
\end{aligned}
$$

where we have set $B_{r}^{-}(0):=\left\{x \in B_{r}(0): x_{N} \leq 0\right\}$. Since $N \geq 2$, we may choose $R^{\prime} \geq 1$ sufficiently large to guarantee that

$$
p c_{p} C_{G S} r^{-\frac{N-1}{2}} \int_{\mathbb{R}^{N}} \psi^{p-1} d x \leq \frac{m_{c, 1}}{4} \int_{B_{1}(0)} \psi^{p} d x, \quad \text { for } r \geq R^{\prime},
$$

and therefore

$$
E_{\infty}^{+}\left(t \psi_{r}\right)-E\left(t \psi_{r}\right) \geq C_{2} e^{-r} t^{p}, \quad \text { for } r \geq R^{\prime}, \quad \text { with } \quad C_{2}:=\frac{\kappa m_{c, 1}}{4} \int_{B_{1}(0)} \psi^{p} d x
$$

Hence the claim follows.

Lemma 4.4 Let $R^{\prime} \geq 1$ be given as in Lemma 4.3. Then there exist $k>0$ with $E\left(k \psi_{r}\right)<0$ for all $r \geq R^{\prime}$. 
Proof Let $k>0$. For $r \geq R^{\prime} \geq 1$ we have, by Lemma 4.3 and since the map $r \mapsto \varepsilon_{r}$ is strictly decreasing by (4.7),

$$
\begin{aligned}
E\left(k \psi_{r}\right) & \leq E_{\infty}^{+}\left(k \psi_{r}\right)=\frac{k^{2}}{2}\left\|\psi_{r}\right\|^{2}-\frac{k^{p+1}}{p+1} \int_{\mathbb{R}_{+}^{N}} \psi_{r}^{p+1} d x \\
& \leq \frac{k^{2}}{2}\|\psi\|_{H^{1}\left(\mathbb{R}^{N}\right)}^{2}-\frac{k^{p+1}}{p+1} \int_{B_{r}(0)}\left(\psi-\varepsilon_{r}\right)^{p+1} d x \\
& \leq \frac{k^{2}}{2}\|\psi\|_{H^{1}\left(\mathbb{R}^{N}\right)}^{2}-\frac{k^{p+1}}{p+1} \int_{B_{1}(0)}\left(\psi-\varepsilon_{1}\right)^{p+1} d x
\end{aligned}
$$

Since $\int_{B_{1}(0)}\left(\psi-\varepsilon_{1}\right)^{p+1} d x>0$ by (4.7), we may choose

$$
k>\left(\frac{(p+1)\|\psi\|_{H^{1}\left(\mathbb{R}^{N}\right)}^{2}}{2 \int_{B_{1}(0)}\left(\psi-\varepsilon_{1}\right)^{p+1} d x}\right)^{\frac{1}{p-1}}
$$

which implies that $E\left(k \psi_{r}\right)<0$ for $r \geq R^{\prime}$, as claimed.

Proof of Proposition 4.1 Let $R^{\prime} \geq 1$ be given by Lemma 4.3, and let $k>0$ be given by Lemma 4.4. For $r \geq R^{\prime}$ and $t \in[0, k]$ we then have, by Lemmas 4.2 and 4.3,

$$
\begin{aligned}
E\left(t \psi_{r}\right) & =E_{\infty}^{+}\left(t \psi_{r}\right)-\left(E_{\infty}^{+}\left(t \psi_{r}\right)-E\left(t \psi_{r}\right)\right) \leq b_{\infty}+e^{-r}\left(t^{p+1} C_{1} r^{-\frac{N-1}{2}}-t^{p} C_{2}\right) \\
& \leq b_{\infty}+e^{-r} t^{p}\left(k C_{1} r^{-\frac{N-1}{2}}-C_{2}\right) .
\end{aligned}
$$

Since $N \geq 2$, we may fix $R \geq R^{\prime}$ with the property that $k C_{1} r^{-\frac{N-1}{2}} \leq \frac{C_{2}}{2}$ for $r \geq R$, which implies that

$$
E\left(t \psi_{r}\right) \leq b_{\infty}-\frac{C_{2}}{2} e^{-r} t^{p}<b_{\infty} \text { for } t \in(0, k], r \geq R
$$

Since also $E(0)=0<b_{\infty}$, we thus obtain that $E\left(t \psi_{r}\right)<b_{\infty}$ for $t \in[0, k], r \geq R$. Moreover, by Lemma 4.4 we have $E\left(k \psi_{r}\right)<0$ for all $r \geq R$ since $R \geq R^{\prime}$. The proof is finished.

\section{The existence result}

We keep using the notation of the introduction and of Sect. 2, which depends on the fixed quantities $c \in\left(0, c_{p}\right)$ and $p \in\left(1,2^{*}-1\right)$. Moreover, we will assume $N \geq 2$ throughout this section, which will allow us to prove the existence of a non-trivial solution to (1.14). This will conclude the proof of Theorem 1.3.

Theorem 5.1 Let $N \geq 2$. Then there exists a non-trivial solution $u \in H_{0}^{1}\left(\mathbb{R}_{+}^{N}\right)$ to (1.14) which, in particular, is a positive solution to (1.11). 
The strategy of the proof is as follows: using the strict inequality (4.9), we will manage to prove the existence of a Cerami sequence whose weak limit is non trivial and thus we will obtain a non trivial solution to (1.14).

Proof of Theorem 5.1 Since the functional $E$ has a mountain pass geometry (see Lemma 3.1), there exists a Cerami sequence for $E$ at the corresponding mountain pass level $b$ defined in (4.1) (see e.g. [10] or [13, Theorem 6, Sect. 1, Chapter IV]), i.e. there exists $\left(u_{n}\right)_{n} \subset H_{0}^{1}\left(\mathbb{R}_{+}^{N}\right)$ such that

$$
E\left(u_{n}\right) \rightarrow b \quad \text { and } \quad\left(1+\left\|u_{n}\right\|\right)\left\|E^{\prime}\left(u_{n}\right)\right\|_{H^{-1}\left(\mathbb{R}_{+}^{N}\right)} \rightarrow 0, \quad \text { as } n \rightarrow \infty .
$$

By Proposition 3.2 we know that $\left(u_{n}\right)_{n}$ is bounded in $H_{0}^{1}\left(\mathbb{R}_{+}^{N}\right)$. Moreover,

$$
\left\|u_{n}^{-}\right\|^{2}=\left\langle E^{\prime}\left(u_{n}\right), u_{n}^{-}\right\rangle \rightarrow 0 \quad \text { as } n \rightarrow \infty
$$

Let $\left(y^{n}\right)_{n} \subset \mathbb{R}_{+}^{N}$ be the sequence of points obtained in Lemma 3.3 applied to $\left(u_{n}\right)_{n}$, i.e., we have

$$
\liminf _{n \rightarrow \infty} \int_{B_{R}\left(y^{n}\right) \cap \mathbb{R}_{+}^{N}} u_{n}^{2} d x \geq \delta \quad \text { for some } \delta>0 .
$$

We split the argument into two steps.

Step 1: There exists $M>0$ such that $y_{N}^{n}=\operatorname{dist}\left(y^{n}, \partial \mathbb{R}_{+}^{N}\right) \leq M$ for all $n \in \mathbb{N}$.

We assume by contradiction that

$$
\lim _{n \rightarrow \infty} y_{N}^{n}=+\infty
$$

Then, let us define, for all $n \in \mathbb{N}, w_{n}:=u_{n}\left(\cdot+y^{n}\right)$. By Lemma 3.3 and (5.1), it follows that

$$
\begin{aligned}
& w_{n} \rightarrow w \text { in } H^{1}\left(\mathbb{R}^{N}\right), \quad w_{n} \rightarrow w \text { in } L_{l o c}^{q}\left(\mathbb{R}^{N}\right) \text { for } 1 \leq q<2^{*}, \quad \text { and } \\
& w_{n} \rightarrow w \text { a.e. in } \mathbb{R}^{N},
\end{aligned}
$$

for some $w \in H^{1}\left(\mathbb{R}^{N}\right)$ with $w \geq 0, w \not \equiv 0$. We also observe that

$$
\begin{aligned}
b+o(1) & =E\left(u_{n}\right)-\frac{1}{2}\left\langle E^{\prime}\left(u_{n}\right), u_{n}\right\rangle=\int_{\mathbb{R}_{+}^{N}} H\left(x, u_{n}(x)\right) d x \\
& =\int_{\left\{x_{N} \geq-y_{N}^{n}\right\}} H\left(x+y_{n}, w_{n}^{+}(x)\right) d x, \quad \text { as } n \rightarrow \infty,
\end{aligned}
$$

with the function $H$ defined in Lemma 2.2 (ii). Next, we note that

$$
H\left(x+y_{n}, w_{n}^{+}(x)\right) \geq 0, \quad \text { for } x \in\left\{x_{N} \geq-y_{N}^{n}\right\},
$$


and

$$
\begin{aligned}
\liminf _{n \rightarrow \infty} H\left(x+y_{n}, w_{n}^{+}(x)\right) \geq & \liminf _{n \rightarrow \infty}\left(\frac{p-1}{2(p+1)}\left[w_{n}^{+}(x)\right]^{p+1}-u_{c}\left(x+y_{n}\right)^{p-1} D_{1, p}\left[w_{n}^{+}(x)\right]^{2}\right. \\
& \left.-u_{c}\left(x+y_{n}\right) 1_{\{p>2\}} D_{2, p}\left[w_{n}^{+}(x)\right]^{p}\right) \\
& =\frac{p-1}{2(p+1)}\left[w^{+}(x)\right]^{p+1} \\
& =\frac{p-1}{2(p+1)} w^{p+1}(x), \quad \text { for } x \in\left\{x_{N} \geq-y_{N}^{n}\right\},
\end{aligned}
$$

by Lemma 2.2 (ii) and (4.10). Thus, (5.5) and Fatou's Lemma imply that

$$
\frac{p-1}{2(p+1)}\|w\|_{L^{p+1}\left(\mathbb{R}^{N}\right)}^{p+1} \leq b .
$$

Next we claim that $w \in \mathcal{K}_{\infty}$, i.e., $w$ is a nontrivial solution of (4.3). To see this, we fix an arbitrary $\varphi \in C_{c}^{\infty}\left(\mathbb{R}^{N}\right)$, and we show that

$$
\int_{\mathbb{R}^{N}}(\nabla w \nabla \varphi+w \varphi) d x=\int_{\mathbb{R}^{N}}\left(w^{+}\right)^{p} \varphi d x
$$

Since (5.3) holds, we have that $\operatorname{supp}(\varphi) \subset\left\{x_{N} \geq-y_{N}^{n}\right\}$ for $n \in \mathbb{N}$ sufficiently large. Hence, for $n \in \mathbb{N}$ large enough, we have that

$$
\begin{aligned}
o(1)= & \left\langle E^{\prime}\left(u_{n}\right), \varphi\left(\cdot-y^{n}\right)\right\rangle \\
= & \int_{\left\{x_{N} \geq-y_{N}^{n}\right\}}\left(\nabla w_{n} \nabla \varphi+w_{n} \varphi\right) d x \\
& -\int_{\left\{x_{N} \geq-y_{N}^{n}\right\}}\left(u_{c}\left(\cdot+y^{n}\right)+w_{n}^{+}\right)^{p} \varphi d x-\int_{\left\{x_{N} \geq-y_{N}^{n}\right\}} u_{c}^{p}\left(\cdot+y^{n}\right) \varphi d x \\
= & \int_{\mathbb{R}^{N}}\left(\nabla w_{n} \nabla \varphi+w_{n} \varphi\right) d x \\
& -\int_{\mathbb{R}^{N}}\left(u_{c}\left(\cdot+y^{n}\right)+w_{n}^{+}\right)^{p} \varphi d x+o(1) \\
= & \int_{\mathbb{R}^{N}}\left(\nabla w_{n} \nabla \varphi+w_{n} \varphi\right) d x-\int_{\mathbb{R}^{N}}\left(w_{n}^{+}\right)^{p} \varphi d x \\
& -\int_{\mathbb{R}^{N}}\left(\left(u_{c}\left(\cdot+y^{n}\right)+w_{n}^{+}\right)^{p}-\left(w_{n}^{+}\right)^{p}\right) \varphi d x+o(1) \\
= & \int_{\mathbb{R}^{N}}\left(\nabla w_{n} \nabla \varphi+w_{n} \varphi\right) d x-\int_{\mathbb{R}^{N}}\left(w_{n}^{+}\right)^{p} \varphi d x+o(1) \\
= & \int_{\mathbb{R}^{N}}(\nabla w \nabla \varphi+w \varphi) d x-\int_{\mathbb{R}^{N}}\left(w^{+}\right)^{p} \varphi d x+o(1), \quad \text { as } n \rightarrow \infty .
\end{aligned}
$$


Hence (5.7) follows, and therefore $w \in \mathcal{K}_{\infty}$. Together with (4.8) and (5.6) it then follows that $b \geq b_{\infty}$, but this contradicts (4.9). Hence, (5.3) cannot happen and Step 1 follows.

\section{Step 2: Conclusion.}

Let us define, for all $n \in \mathbb{N}, v_{n}:=u_{n}\left(\cdot+\xi_{n}\right)$ with $\xi_{n}:=\left(y_{1}^{n}, \ldots, y_{N-1}^{n}, 0\right)$ and observe that, after passing to a subsequence

$v_{n} \rightarrow v$ in $H_{0}^{1}\left(\mathbb{R}_{+}^{N}\right), \quad v_{n} \rightarrow v$ in $L_{l o c}^{q}\left(\mathbb{R}_{+}^{N}\right)$ for $1 \leq q<2^{*} \quad$ and $\quad v_{n} \rightarrow v$ a.e. in $\mathbb{R}_{+}^{N}$,

for some $v \in H_{0}^{1}\left(\mathbb{R}_{+}^{N}\right)$. Also, note that $\left(v_{n}\right)_{n} \subset H_{0}^{1}\left(\mathbb{R}_{+}^{N}\right)$ is a Cerami sequence for $E$ at level $b$. Hence, if $v \neq \equiv 0$, we will have that $v$ is a non-trivial solution to (1.14). Since $v_{n} \rightarrow v$ in $L_{l o c}^{q}\left(\mathbb{R}_{+}^{N}\right)$ and $y_{N}^{n} \leq M$ for all $n \in \mathbb{N}$, the lower integral bound (5.2) implies that $v \not \equiv 0$, and the result follows.

Proof of Theorem 1.3 (Completed) Let $u \in H_{0}^{1}\left(\mathbb{R}_{+}^{N}\right)$ be the non-negative and nontrivial solution to (1.11) obtained in Theorem 5.1. By standard elliptic regularity we have that $u \in C^{2}\left(\mathbb{R}_{+}^{N}\right) \cap C\left(\overline{\mathbb{R}_{+}^{N}}\right) \cap L^{\infty}\left(\mathbb{R}_{+}^{N}\right)$ and $v:=u_{c}+u$ is a bounded positive solution to (1.3)-(1.4) of the form (1.8).

Remark 5.1 As explained in the introduction, Theorem 1.2 (i) and Corollary 1.4 are direct consequences of Theorem 1.3.

In the remaining of this section we prove Proposition 1.5. Let us first state a technical lemma due to Poláčik, Quittner and Souplet that will be key to prove this result.

Lemma 5.2 (Particular case of [29, Lemma 5.1]) Let $(X, d)$ be a complete metric space and let $M: X \rightarrow(0,+\infty)$ be continuous. For any $\delta<\sup _{X} M$ and any $k>0$ there exists $y \in X$ such that

- $M(y) \geq \delta$.

- $M(z) \leq 2 M(y)$ for all $z \in X$ with $d(z, y) \leq \frac{k}{M(y)}$.

The following proof is inspired by [12, Lemma 2.5].

Proof of Proposition 1.5 We assume by contradiction that there exists $v \in C^{2}\left(\mathbb{R}_{+}^{N}\right) \cap$ $C\left(\overline{\mathbb{R}_{+}^{N}}\right)$ unbounded solving (1.3)-(1.4). By Lemma 5.2 applied with $X=\overline{\mathbb{R}_{+}^{N}}$ and $M=v^{\frac{p-1}{2}}$, there exits a sequence $\left(y^{k}\right)_{k} \subset \mathbb{R}_{+}^{N}$ such that

$$
\begin{aligned}
& M\left(y^{k}\right) \rightarrow \infty, \quad \text { as } k \rightarrow \infty, \\
& M(z) \leq 2 M\left(y^{k}\right), \quad \text { for all } z \in \mathbb{R}_{+}^{N} \text { with } d\left(z, y^{k}\right) \leq \frac{k}{M\left(y^{k}\right)} \text { and all } k \in \mathbb{N} .
\end{aligned}
$$

Note that, without loss of generality, we can suppose that $M\left(y^{k}\right) \geq 1$ for all $k \in \mathbb{N}$. We then define, for all $k \in \mathbb{N}, d_{k}:=y_{N}^{k} M\left(y^{k}\right)$, the half-space $H_{k}:=\left\{x \in \mathbb{R}_{+}^{N}\right.$ : $\left.x_{N}>-d_{k}\right\}$ and

$$
v^{k}: H_{k} \rightarrow \mathbb{R}_{+}^{N} \quad \text { given by } \quad v^{k}(z):=\frac{1}{v\left(y^{k}\right)} v\left(y^{k}+\frac{z}{M\left(y^{k}\right)}\right) .
$$


Note that, for all $k \in \mathbb{N}, v^{k}$ is a positive solution to

$$
\left\{\begin{aligned}
-\Delta v^{k}+\frac{1}{M^{2}\left(y^{k}\right)} v^{k} & =\left(v^{k}\right)^{p}, & & \text { in } H_{k}, \\
v^{k} & =\frac{c}{M^{\frac{2}{p-1}}\left(y^{k}\right)}, & & \text { on } \partial H_{k},
\end{aligned}\right.
$$

and, by its definition and (5.11), it satisfies

$$
v^{k}(0)=1 \quad \text { and } \quad v^{k}(z) \leq 2^{\frac{2}{q-1}} \quad \text { for all } z \in H_{k} \cap B_{k}(0)
$$

We now consider two cases separately.

Case 1: $d_{k} \rightarrow \infty$ as $k \rightarrow \infty$.

Using standard $L^{q}$ estimates (see e.g. [18, Chapter 9]), (5.12) and (5.13), we get (taking a subsequence if necessary) that $\left(v^{k}\right)_{k}$ is locally $W^{2, q}$-bounded in $\mathbb{R}^{N}$ for arbitrarily large $q<+\infty$. Hence, up to a subsequence, $v^{k} \rightarrow \bar{v}$ in $C_{l o c}^{1}\left(\mathbb{R}^{N}\right)$, where $\bar{v} \in C^{1}\left(\mathbb{R}^{N}\right)$ is a non-trivial positive solution to

$$
\Delta \bar{v}+\bar{v}^{p}=0, \quad \text { in } \mathbb{R}^{N}
$$

By (5.13) we infer that $\bar{v}$ is bounded in $\mathbb{R}^{N}$ with $\bar{v}(0)=1$. Hence $\bar{v} \in C^{2}\left(\mathbb{R}^{N}\right)$ by standard elliptic regularity. Then, since by [17, Theorem 1.2] we know that the only $C^{2}\left(\mathbb{R}^{N}\right)$ non-negative solution to $(5.14)$ is $\bar{v} \equiv 0$, we obtain a contradiction and deduce that Case 1 cannot happen.

Case 2: $d_{k} \rightarrow d \geq 0$ as $k \rightarrow \infty$.

Let us define, for all $k \in \mathbb{N}$,

$$
w^{k}: \mathbb{R}_{+}^{N} \rightarrow \mathbb{R} \quad \text { as } \quad w^{k}(z)=v^{k}\left(z-d_{k} e_{N}\right)
$$

where $e_{N}:=(0, \ldots, 0,1)$ is the $N$-th coordinate vector. Note that, for all $k \in \mathbb{N}, w^{k}$ is a positive solution to

$$
\left\{\begin{array}{rlrl}
-\Delta w^{k}+\frac{1}{M^{2}\left(y^{k}\right)} w^{k} & =\left(w^{k}\right)^{p}, & & \text { in } \mathbb{R}_{+}^{N}, \\
w^{k}=\frac{c}{M^{\frac{2}{p-1}}\left(y^{k}\right)}, & & \text { on } \partial \mathbb{R}_{+}^{N},
\end{array}\right.
$$

and satisfies

$$
w^{k}\left(d_{k} e_{N}\right)=1 \quad \text { and } \quad w^{k}(z) \leq 2^{\frac{2}{p-1}} \quad \text { for all } z \in \overline{\mathbb{R}_{+}^{N}} \cap B_{k}\left(d_{k} e_{N}\right) .
$$

Now, arguing as in the proof of [18, Theorem 9.13] (with auxiliary functions $\varphi^{k}=w^{k}-$ $c M^{-\frac{2}{p-1}}\left(y^{k}\right)$ ) and taking into account (5.16) and (5.17), we get (taking a subsequence 
if necessary) that $\left(w^{k}\right)_{k}$ is locally $W^{2, q}$-bounded in $\overline{\mathbb{R}_{+}^{N}}$ for arbitrarily large $q<+\infty$ and therefore also locally $C^{1, \beta}$-bounded in $\overline{\mathbb{R}_{+}^{N}}$ for all $\beta \in(0,1)$. In particular, $\left|\nabla w^{k}\right|$ remains bounded pointwise independently of $k$ in a neighbourhood of the origin. Taking into account (5.10), the boundary conditions in (5.16) and (5.17), we infer that $d=\lim _{k \rightarrow \infty} d_{k}>0$. Hence, up to a subsequence, $w^{k} \rightarrow \bar{w}$ in $C_{\text {loc }}^{1}\left(\mathbb{R}_{+}^{N}\right)$ with $\bar{w} \in C^{1}\left(\mathbb{R}_{+}^{N}\right)$ a non-trivial positive solution to

$$
\left\{\begin{aligned}
\Delta \bar{w}+\bar{w}^{p} & =0, & & \text { in } \mathbb{R}_{+}^{N}, \\
\bar{w} & =0, & & \text { on } \partial \mathbb{R}_{+}^{N} .
\end{aligned}\right.
$$

By (5.17) we have that $\bar{w}$ is bounded with $\bar{w}\left(d e_{N}\right)=1$. Hence $\bar{w} \in C^{2}\left(\mathbb{R}^{N}\right) \cap C\left(\overline{\mathbb{R}_{+}^{N}}\right)$ by standard elliptic regularity. Then, since by [17, Theorem 1.3] we know that the only $C^{2}\left(\mathbb{R}_{+}^{N}\right) \cap C\left(\overline{\mathbb{R}_{+}^{N}}\right)$ non-negative solution to $(5.18)$ is $\bar{w} \equiv 0$, we obtain a contradiction and deduce that Case 2 cannot happen either. Hence, the result follows.

\section{The non-existence result}

In this section we prove Part (ii) of Theorem 1.2, which is concerned with the nonexistence of bounded positive solutions to (1.3)-(1.4) in the case $c>c_{p}$. Recall that

$$
w_{0}(t)=c_{p}\left[\cosh \left(\frac{p-1}{2} t\right)\right]^{-\frac{2}{p-1}},
$$

is the unique even non-trivial positive solution to (1.5). Throughout this section, we will use the following notation. We define $v_{0}: \mathbb{R}^{N} \rightarrow \mathbb{R}$ as

$$
v_{0}(x)=w_{0}\left(x_{N}\right), \quad \text { for } x \in \mathbb{R}^{N} \text {. }
$$

Also, recall that for a bounded positive solution to (1.3)-(1.4), we mean a function $v \in C^{2}\left(\mathbb{R}_{+}^{N}\right) \cap C\left(\overline{\mathbb{R}_{+}^{N}}\right) \cap L^{\infty}\left(\mathbb{R}_{+}^{N}\right)$, positive, satisfying (1.3) in the pointwise sense and such that (1.4) holds.

Theorem 6.1 For $N \geq 1, p>1$ and $c>c_{p}$, there are no bounded positive solutions to (1.3)-(1.4).

Proof Let us fix an arbitrary $c>c_{p}$. We assume by contradiction that there exists a bounded positive solution $v$ to (1.3)-(1.4) and we define, for all $t \in \mathbb{R}, v_{t}:=v_{0}\left(\cdot+t e_{N}\right)$ where $v_{0}$ is given in $(6.1)$ and $e_{N}=(0, \ldots, 0,1)$ is the $N$-th coordinate vector. We split the proof into three steps.

Step 1: There exists $t_{0}>0$ such that $v>v_{t}$ in $\overline{\mathbb{R}_{+}^{N}}$ for all $t \geq t_{0}$.

First of all, fixed an arbitrary $x \in \overline{\mathbb{R}_{+}^{N}}$, observe that

$$
v_{t}(x) \rightarrow 0, \quad \text { as } t \rightarrow \infty, \quad \text { and } \quad v_{t_{1}}(x)>v_{t_{2}}(x), \quad \text { for all } 0<t_{1}<t_{2}
$$


Hence, there exists $t_{0}>0$ such that, for all $t \geq t_{0}$,

$$
v_{t} \leq\left(\frac{1}{2 p}\right)^{\frac{1}{p-1}} \text { in } \overline{\mathbb{R}_{+}^{N}} .
$$

We fix $t_{0}>0$ such that (6.2) holds and we are going to prove the Step 1 for this $t_{0}$. To that end, we fix an arbitrary $t \geq t_{0}>0$. First, we are going to prove that $v \geq v_{t}$ in $\overline{\mathbb{R}_{+}^{N}}$. Since $c>c_{p} \geq \max _{x \in \mathbb{R}} v_{t}(x)$, we have that

$$
\left\{\begin{aligned}
-\Delta\left(v-v_{t}\right)+\left(v-v_{t}\right) & =|v|^{p-1} v-\left|v_{t}\right|^{p-1} v_{t}, & & \text { in } \mathbb{R}_{+}^{N}, \\
v-v_{t} & >0, & & \text { on } \partial \mathbb{R}_{+}^{N},
\end{aligned}\right.
$$

or equivalently

$$
\left\{\begin{aligned}
-\Delta\left(v-v_{t}\right)+c_{t}(x)\left(v-v_{t}\right) & =0, \\
v-v_{t} & >0, \quad \text { in } \mathbb{R}_{+}^{N},
\end{aligned}\right.
$$

where

$$
c_{t}(x):= \begin{cases}1-\frac{(v(x))^{p}-\left(v_{t}(x)\right)^{p}}{v(x)-v_{t}(x)}, & \text { if } v(x)-v_{t}(x) \neq 0, \\ 1, & \text { if } v(x)-v_{t}(x)=0 .\end{cases}
$$

We assume by contradiction that

$$
\left\{x \in \mathbb{R}_{+}^{N}: v(x)<v_{t}(x)\right\} \neq \emptyset
$$

Then, using the mean value theorem and (6.2), we deduce that, for all $x \in\left\{x \in \mathbb{R}_{+}^{N}\right.$ : $\left.v(x)<v_{t}(x)\right\}$

$$
c_{t}(x) \geq 1-p\left(v_{t}(x)\right)^{p-1} \geq \frac{1}{2} .
$$

Hence, in each connected component $D$ of $\left\{x \in \mathbb{R}_{+}^{N}: v(x)<v_{t}(x)\right\}$ we have that

$$
\left\{\begin{aligned}
-\Delta\left(v-v_{t}\right)+c_{t}(x)\left(v-v_{t}\right) & =0, \quad \text { in } D, \\
v-v_{t} & =0, \quad \text { on } \partial D,
\end{aligned}\right.
$$

with $c_{t}$ satisfying (6.7). Then, applying the weak maximum principle [5, Lemma 2.1], we obtain that $v \geq v_{t}$ in $\bar{D}$ which contradicts the fact that $D \subset\left\{x \in \mathbb{R}_{+}^{N}: v(x)<\right.$ $\left.v_{t}(x)\right\}$. Hence, we conclude that $\left\{x \in \mathbb{R}_{+}^{N}: v(x)<v_{t}(x)\right\}=\emptyset$ and so, that $v \geq v_{t}$ in $\mathbb{R}_{+}^{N}$. Having this at hand and substituting in (6.3), we deduce that

$$
\left\{\begin{aligned}
-\Delta\left(v-v_{t}\right)+\left(v-v_{t}\right) & \geq 0, \\
v-v_{t} & >0, \quad \text { in } \mathbb{R}_{+}^{N},
\end{aligned}\right.
$$


and so, the Step 1 follows from the strong maximum principle and the fact that $t \geq t_{0}$ is arbitrary.

Step 2: $v>v_{t}$ in $\overline{\mathbb{R}_{+}^{N}}$ for all $t \in \mathbb{R}$.

Note that, if we prove that $v \geq v_{t}$ in $\mathbb{R}_{+}^{N}$ for all $t \in \mathbb{R}$, then the claim follows from the Strong Maximum principle. Also, by the Step 1, we know that

$$
\left\{t \in \mathbb{R}: v \geq v_{s} \text { in } \mathbb{R}_{+}^{N} \text { for all } s \geq t\right\} \neq \emptyset .
$$

Hence, we can define

$$
t_{\star}:=\inf \left\{t \in \mathbb{R}: v \geq v_{s} \text { in } \mathbb{R}_{+}^{N} \text { for all } s \geq t\right\} \in\left[-\infty, t_{0}\right] .
$$

We argue by contradiction and suppose that $t_{\star}>-\infty$. First note that, by continuity, $v \geq v_{t_{\star}}$ in $\mathbb{R}_{+}^{N}$. Also, $t_{\star}>-\infty$ implies the existence of $M>0$ such that

$$
v_{t}\left(x^{\prime}, x_{N}\right) \leq\left(\frac{1}{2 p}\right)^{\frac{1}{p-1}}, \quad \text { for all } t \in\left[t_{\star}-1, t_{\star}\right], x^{\prime} \in \mathbb{R}^{N-1} \text { and } x_{N} \geq M
$$

We now consider separately two cases.

Case 1: $\inf _{x \in \mathbb{R}^{N-1} \times[0, M]}\left(v-v_{t_{\star}}\right)=: \delta_{M}>0$.

First, taking into account that $\left\|w_{0}^{\prime}\right\|_{L^{\infty}(\mathbb{R})} \leq c_{p}$, we infer that, for all $t \leq t_{\star}$ and $x \in \mathbb{R}^{N-1} \times[0, M]$,

$$
\begin{aligned}
v\left(x^{\prime}, x_{N}\right)-v_{t}\left(x^{\prime}, x_{N}\right) & =v\left(x^{\prime}, x_{N}\right)-v_{t_{\star}}\left(x^{\prime}, x_{N}\right)+\left(v_{t_{\star}}\left(x^{\prime}, x_{N}\right)-v_{t}\left(x^{\prime}, x_{N}\right)\right) \\
& \geq \delta_{M}-\left|w_{0}\left(x_{N}+t_{\star}\right)-w_{0}\left(x_{N}+t\right)\right| \\
& \geq \delta_{M}-c_{p}\left|t_{\star}-t\right| .
\end{aligned}
$$

Hence, there exists $\eta_{0} \in(0,1)$ such that, for all $t_{\star} \geq t \geq t_{\star}-\eta_{0}$,

$$
v\left(x^{\prime}, x_{N}\right)-v_{t}\left(x^{\prime}, x_{N}\right)>0, \quad \text { for all } x^{\prime} \in \mathbb{R}^{N-1} \text { and } x_{N} \in[0, M]
$$

In particular, if we define $\Sigma_{M}:=\left\{x \in \mathbb{R}^{N}: x_{N}>M\right\}$, we have

$$
v-v_{t}>0, \quad \text { on } \partial \Sigma_{M}, \quad \text { for all } t \in\left[t_{\star}-\eta_{0}, t_{\star}\right] \text {. }
$$

Next, we are going to prove that, for all $t \in\left[t_{\star}-\eta_{0}, t_{\star}\right]$, it follows $v \geq v_{t}$ in $\Sigma_{M}$. To that end, we fix an arbitrary $t \in\left[t_{\star}-\eta_{0}, t_{\star}\right]$. Arguing as in Step 1 , we have that

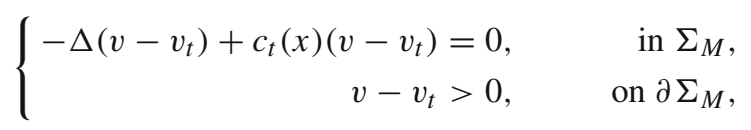


where

$$
c_{t}(x):= \begin{cases}1-\frac{(v(x))^{p}-\left(v_{t}(x)\right)^{p}}{v(x)-v_{t}(x)}, & \text { if } v(x)-v_{t}(x) \neq 0 \\ 1, & \text { if } v(x)-v_{t}(x)=0 .\end{cases}
$$

We assume by contradiction that

$$
\left\{x \in \Sigma_{M}: v(x)<v_{t}(x)\right\} \neq \emptyset .
$$

Then, using the mean value theorem and (6.11), we deduce that, for all $x \in\left\{x \in \Sigma_{M}\right.$ : $\left.v(x)<v_{t}(x)\right\}$

$$
c_{t}(x) \geq 1-p\left(v_{t}(x)\right)^{p-1} \geq \frac{1}{2} .
$$

Hence, in each connected component $D$ of $\left\{x \in \Sigma_{M}: v(x)<v_{t}(x)\right\}$ we have that

$$
\left\{\begin{aligned}
-\Delta\left(v-v_{t}\right)+c_{t}(x)\left(v-v_{t}\right) & =0, \\
v-v_{t} & =0, \quad \text { in } D,
\end{aligned}\right.
$$

with $c_{t}$ satisfying (6.16). Then, applying the weak maximum principle [5, Lemma 2.1], we obtain that $v \geq v_{t}$ in $\bar{D}$ which contradicts the fact that $D \subset\left\{x \in \Sigma_{M}\right.$ : $\left.v(x)<v_{t}(x)\right\}$. Hence, we conclude that $\left\{x \in \Sigma_{M}: v(x)<v_{t}(x)\right\}=\emptyset$ and so, that $v \geq v_{t}$ in $\Sigma_{M}$. Taking into account (6.12), we infer that, for all $\eta \in\left[0, \eta_{0}\right], v \geq v_{t_{\star}-\eta}$ in $\mathbb{R}_{+}^{N}$. This is in contradiction with the definition of $t_{\star}$. Hence, Case 1 cannot happen.

Case 2: $\inf _{x \in \mathbb{R}^{N-1} \times[0, M]}\left(v-v_{t_{\star}}\right)=0$.

In this case there exists a sequence of points $\left(x^{n}\right)_{n} \subset \mathbb{R}^{N-1} \times[0, M]$ such that

$$
v\left(x^{n}\right)-v_{t_{\star}}\left(x^{n}\right) \rightarrow 0, \quad \text { as } n \rightarrow \infty .
$$

Up to a subsequence, it follows that $x_{N}^{n} \rightarrow \bar{x}_{N}$ for some $\bar{x}_{N} \in[0, M]$. We define then

$$
v^{n}(x)=v\left(x^{\prime}+\left(x^{n}\right)^{\prime}, x_{N}\right), \quad \text { for all } n \in \mathbb{N},
$$

and, for all $n \in \mathbb{N}$, we have $v^{n} \geq v_{t_{\star}}$ in $\mathbb{R}_{+}^{N}$ and

$$
\left\{\begin{aligned}
-\Delta v^{n}+v^{n} & =\left(v^{n}\right)^{p}, & & \text { in } \mathbb{R}_{+}^{N}, \\
v^{n} & =c, & & \text { on } \partial \mathbb{R}_{+}^{N} .
\end{aligned}\right.
$$

Moreover, for all $n \in \mathbb{N}$, it follows that

$$
\left\{\begin{array}{r}
-\Delta\left(v^{n}-v_{t_{\star}}\right)+\left(v^{n}-v_{t_{\star}}\right) \geq 0, \quad \text { in } \mathbb{R}_{+}^{N}, \\
v^{n}-v_{t_{\star}}>0, \quad \text { on } \partial \mathbb{R}_{+}^{N},
\end{array}\right.
$$


and so, by the Strong Maximum principle, we have that

$$
v^{n}-v_{t_{\star}}>0, \quad \text { in } \overline{\mathbb{R}_{+}^{N}}, \quad \text { for all } n \in \mathbb{N}
$$

Now, arguing as in [15, Proof of Theorem 2.1, Step 1], we deduce that the sequence $\left(v^{n}\right)_{n}$ admits a subsequence, still denoted by $\left(v^{n}\right)_{n}$, converging to a function $\bar{v}$ in $C_{\text {loc }}^{2}\left(\mathbb{R}_{+}^{N}\right)$. This function $\bar{v}$ still solves

$$
\left\{\begin{aligned}
-\Delta \bar{v}+\bar{v} & =\bar{v}^{p}, & \text { in } \mathbb{R}_{+}^{N}, \\
\bar{v} & =c, & \text { on } \partial \mathbb{R}_{+}^{N},
\end{aligned}\right.
$$

and satisfies $\bar{v} \geq v_{t_{\star}}$, in $\mathbb{R}_{+}^{N}$, and

$$
\bar{v}\left(0^{\prime}, \bar{x}_{N}\right)=v_{t_{\star}}\left(0^{\prime}, \bar{x}_{N}\right) .
$$

Note that (6.19) and $\bar{v}=c>c_{p} \geq v_{t_{\star}}$ on $\partial \mathbb{R}_{+}^{N}$ imply $\bar{x}_{N}>0$. Since $\bar{v} \geq v_{t_{\star}}$ in $\mathbb{R}_{+}^{N}$, we have

$$
\left\{\begin{aligned}
-\Delta\left(\bar{v}-v_{t_{\star}}\right)+\left(\bar{v}-v_{t_{\star}}\right) & \geq 0, \\
\bar{v}-v_{t_{\star}} & >0, \quad \text { in } \mathbb{R}_{+}^{N},
\end{aligned}\right.
$$

Hence, by the Strong Maximum principle, it follows that $\bar{v}>v_{t_{\star}}$ in $\mathbb{R}_{+}^{N}$ which gives a contradiction with (6.19). Case 2 cannot happen either and hence the Step 2 follows.

\section{Step 3: Conclusion.}

Observe that $v>v_{t}$ in $\mathbb{R}_{+}^{N}$ for all $t \in \mathbb{R}$ implies that $v \geq v_{0}(0)=c_{p}$ in $\mathbb{R}_{+}^{N}$. This gives a contradiction with (1.4) and so the proof is complete.

Acknowledgements The authors wish to thank the anonymous referees for their valuable comments and corrections. Part of this work was done while the first author was visiting the Goethe-Universität Frankfurt. He wishes to thank his hosts for the warm hospitality and the financial support.

Funding Open Access funding enabled and organized by Projekt DEAL

Data Availability Statement This article has no additional data.

\section{Compliance with ethical standards}

Conflict of interest On behalf of all authors, the corresponding author states that there is no conflict of interest.

Open Access This article is licensed under a Creative Commons Attribution 4.0 International License, which permits use, sharing, adaptation, distribution and reproduction in any medium or format, as long as you give appropriate credit to the original author(s) and the source, provide a link to the Creative Commons licence, and indicate if changes were made. The images or other third party material in this article are included in the article's Creative Commons licence, unless indicated otherwise in a credit line to the material. If material is not included in the article's Creative Commons licence and your intended use is not permitted by statutory regulation or exceeds the permitted use, you will need to obtain permission directly from the copyright holder. To view a copy of this licence, visit http://creativecommons.org/licenses/by/4.0/. 


\section{References}

1. Ao, W., Musso, M., Pacard, F., Wei, J.: Solutions without any symmetry for semilinear elliptic problems. J. Funct. Anal. 270(3), 884-956 (2016)

2. Bartsch, T., Willem, M.: Infinitely many nonradial solutions of a Euclidean scalar field equation. J. Funct. Anal. 117(2), 447-460 (1993)

3. Bartsch, T., Willem, M.: Infinitely many radial solutions of a semilinear elliptic problem on $R^{N}$. Arch. Ration. Mech. Anal. 124(3), 261-276 (1993)

4. Berestycki, H., Caffarelli, L., Nirenberg, L.: Further qualitative properties for elliptic equations in unbounded domains. Ann. Scuola Norm. Sup. Pisa Cl. Sci. (4) 25(1-2), 69-94 (1997)

5. Berestycki, H., Caffarelli, L.A., Nirenberg, L.: Monotonicity for elliptic equations in unbounded Lipschitz domains. Commun. Pure Appl. Math. 50(11), 1089-1111 (1997)

6. Berestycki, H., Gallouët, T., Kavian, O.: Équations de champs scalaires euclidiens non linéaires dans le plan. C. R. Acad. Sci. Paris Sér. I Math. 297(5), 307-310 (1983)

7. Berestycki, H., Hamel, F., Monneau, R.: One-dimensional symmetry of bounded entire solutions of some elliptic equations. Duke Math. J. 103(3), 375-396 (2000)

8. Berestycki, H., Lions, P.-L.: Nonlinear scalar field equations I. Existence of a ground state. Arch. Ration. Mech. Anal. 82(4), 313-345 (1983)

9. Berestycki, H., Lions, P.-L.: Nonlinear scalar field equations. II. Existence of infinitely many solutions. Arch. Ration. Mech. Anal. 82(4), 347-375 (1983)

10. Cerami, G.: Un criterio di esistenza per i punti critici su varietà illimitate. Istit. Lomb. Accad. Sci. Lett. Rend. A 112(2), 332-336 (1978)

11. Dancer, E.N.: New solutions of equations on $\boldsymbol{R}^{n}$. Ann. Scuola Norm. Sup. Pisa Cl. Sci. (4) 30(3-4), 535-563 (2002)

12. Dancer, E.N., Weth, T.: Liouville-type results for non-cooperative elliptic systems in a half-space. J. Lond. Math. Soc. (2) 86(1), 111-128 (2012)

13. Ekeland, I.: Convexity Methods in Hamiltonian Mechanics. Springer-Verlag, Berlin (1990)

14. Esteban, M.J., Lions, P.-L.: Existence and nonexistence results for semilinear elliptic problems in unbounded domains. Proc. R. Soc. Edinb. Sect. A 93(1-2):1-14 (1982/83)

15. Farina, A.: Rigidity and one-dimensional symmetry for semilinear elliptic equations in the whole of $\mathbb{R}^{N}$ and in half spaces. Adv. Math. Sci. Appl. 13(1), 65-82 (2003)

16. Gidas, B., Ni, W.M., Nirenberg, L.: Symmetry of positive solutions of nonlinear elliptic equations in $R^{n}$. Adv. Math. Suppl. Stud. 7, 369-402 (1981)

17. Gidas, B., Spruck, J.: A priori bounds for positive solutions of nonlinear elliptic equations. Commun. Partial Differ. Equ. 6(8), 883-901 (1981)

18. Gilbarg, D., Trudinger, N.S.: Elliptic Partial Differential Equations of Second Order. Springer-Verlag, Berlin (2001)

19. Gomez, D., Wei, J.: Multi-spike patterns in the Gierer-Meinhardt system with a non-zero activator boundary flux. Preprint, arXiv:abs/2008.04533 (2020)

20. Jameson, G.J.O.: Inequalities comparing $(a+b)^{p}-a^{p}-b^{p}$ and $a^{p-1} b+a b^{p-1}$. Elem. Math. 69(3), 156-161 (2014)

21. Jeanjean, L.: On the existence of bounded Palais-Smale sequences and application to a LandesmanLazer-type problem set on $R^{N}$. Proc. R. Soc. Edinb. Sect. A 129(4), 787-809 (1999)

22. Jeanjean, L., Tanaka, K.: A note on a mountain pass characterization of least energy solutions. Adv. Nonlinear Stud. 3(4), 445-455 (2003)

23. Krause, A.L., Klika, V., Maini, P.K., Headon, D., Gaffney, E.A.: Isolating patterns in open reactiondiffusion systems. Preprint, arXiv:abs/2009.13114 (2020)

24. Kwong, M.K.: Uniqueness of positive solutions of $\Delta u-u+u^{p}=0$ in $R^{n}$. Arch. Ration. Mech. Anal. 105(3), 243-266 (1989)

25. Lions, P.-L.: The concentration-compactness principle in the calculus of variations. The locally compact case. II. Ann. Inst. H. Poincaré Anal. Non Linéaire 1(4), 223-283 (1984)

26. Liu, Y., Wei, J.: On the Helmholtz equation and Dancer's-type entire solutions for nonlinear elliptic equations. Proc. Am. Math. Soc. 147(3), 1135-1148 (2019)

27. Lorca, S., Ubilla, P.: Symmetric and nonsymmetric solutions for an elliptic equation on $\mathbb{R}^{N}$. Nonlinear Anal. 58(7-8), 961-968 (2004)

28. Musso, M., Pacard, F., Wei, J.: Finite-energy sign-changing solutions with dihedral symmetry for the stationary nonlinear Schrödinger equation. J. Eur. Math. Soc. (JEMS) 14(6), 1923-1953 (2012) 
29. Poláčik, P., Quittner, P., Souplet, P.: Singularity and decay estimates in superlinear problems via Liouville-type theorems. I. Elliptic equations and systems. Duke Math. J. 139(3), 555-579 (2007)

30. Rabinowitz, P.H.: On a class of nonlinear Schrödinger equations. Z. Angew. Math. Phys. 43(2), 270291 (1992)

31. Strauss, W.A.: Existence of solitary waves in higher dimensions. Commun. Math. Phys. 55(2), 149-162 (1977)

32. Struwe, M.: Variational Methods, Applications to Nonlinear Partial Differential Equations and Hamiltonian Systems, vol. 34, 4th edn. Springer-Verlag, Berlin (2008)

33. Stuart, C.A.: Bifurcation from the essential spectrum. In: Matzeu, M., Vignoli, A. (eds.) Topological Nonlinear Analysis, II (Frascati, 1995) of Progress in Nonlinear Differential Equations, vol. 27, pp. 397-443. Birkhäuser Boston Inc., Boston, MA (1997)

34. Willem, M.: Minimax Theorems. Birkhäuser Boston Inc., Boston, MA (1996)

Publisher's Note Springer Nature remains neutral with regard to jurisdictional claims in published maps and institutional affiliations. 